\title{
NuSTAR Observation of Energy Release in 11 Solar Microflares
}

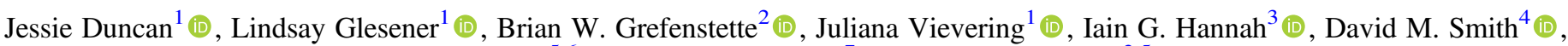 \\ Säm Krucker, ${ }^{5,6}$, Stephen M. White ${ }^{7}$ (D), and Hugh Hudson ${ }^{3,5}$ (D) \\ ${ }^{1}$ University of Minnesota, Minneapolis, MN, USA \\ ${ }^{2}$ California Institute of Technology, Pasadena, CA, USA \\ ${ }^{3}$ University of Glasgow, Glasgow, UK \\ ${ }^{4}$ University of California at Santa Cruz, Santa Cruz, CA, USA \\ ${ }^{5}$ University of California at Berkeley, Berkeley, CA, USA \\ ${ }^{6}$ University of Applied Sciences and Arts Northwestern Switzerland, Windisch, Switzerland \\ 7 Air Force Research Laboratory, Albuquerque, NM, USA \\ Received 2020 August 28; revised 2020 October 22; accepted 2020 November 11; published 2021 February 9
}

\begin{abstract}
Solar flares are explosive releases of magnetic energy. Hard X-ray (HXR) flare emission originates from both hot (millions of Kelvin) plasma and nonthermal accelerated particles, giving insight into flare energy release. The Nuclear Spectroscopic Telescope ARray (NuSTAR) utilizes direct-focusing optics to attain much higher sensitivity in the HXR range than that of previous indirect imagers. This paper presents 11 NuSTAR microflares from two active regions (AR 12671 on 2017 August 21 and AR 12712 on 2018 May 29). The temporal, spatial, and energetic properties of each are discussed in context with previously published HXR brightenings. They are seen to display several "large flare" properties, such as impulsive time profiles and earlier peak times in higher-energy HXRs. For two events where the active region background could be removed, microflare emission did not display spatial complexity; differing NuSTAR energy ranges had equivalent emission centroids. Finally, spectral fitting showed a high-energy excess over a single thermal model in all events. This excess was consistent with additional higher-temperature plasma volumes in 10/11 microflares and only with an accelerated particle distribution in the last. Previous NuSTAR studies focused on one or a few microflares at a time, making this the first to collectively examine a sizable number of events. Additionally, this paper introduces an observed variation in the NuSTAR gain unique to the extremely low livetime $(<1 \%)$ regime and establishes a correction method to be used in future NuSTAR solar spectral analysis.
\end{abstract}

Unified Astronomy Thesaurus concepts: The Sun (1693); Solar flare spectra (1982); Solar x-ray flares (1816); Non-thermal radiation sources (1119); Solar flares (1496); Solar physics (1476)

\section{Introduction}

Solar flares are dramatic manifestations of change in the magnetic structure of the solar corona. They have been observed across over 7 orders of magnitude in estimated Geostationary Operational Environmental Satellite (GOES) soft X-ray (SXR) flux. The accepted model of flare production involves energy released by magnetic reconnection (e.g., Benz 2016). During this process, particles are accelerated to high energies by dynamic fields and emit bremsstrahlung radiation through interactions with ambient coronal plasma (e.g., Brown 1971).

In addition to this nonthermal emission, flares also show significant thermal emission from plasma heated to high temperatures as a result of various mechanisms of energy release. Both nonthermal and thermal emission from the hottest flare plasma (millions of Kelvin) are evident in the hard X-ray (HXR) band, with nonthermal emission dominating at the highest energies (Dennis et al. 2011).

RHESSI operated from 2002 to 2018 and allowed for extensive investigation of large flares using an indirect Fourier imaging method to observe from $3 \mathrm{keV}$ to $17 \mathrm{MeV}$ (Lin et al. 2002). In addition, RHESSI was used for statistical HXR studies of GOES A- and B-class microflares (Christe et al. 2008; Hannah et al. 2008, 2011). However, the large detector volume required by RHESSI's imaging method caused a high background that limited the instrument's sensitivity to fainter events.

In recent years, the Nuclear Spectroscopic Telescope ARray (NuSTAR) satellite mission and the Focusing Optics X-ray
Solar Imager (FOXSI) rocket campaigns have demonstrated the significantly greater sensitivity possible with direct-focusing HXR optics (Harrison et al. 2013; Glesener et al. 2016). These instruments provide an unprecedented opportunity for analysis of microflares, events with an energy content estimated to be around 6 orders of magnitude less than that of the brightest solar flares. NuSTAR and FOXSI are capable of observing the very faintest A-class events, as well as brightenings that are too faint to be observed by GOES.

Flare occurrence rate is inversely related to magnitude, with fainter events observed far more frequently than brighter ones (e.g., Hannah et al. 2011). Because of this, a large ensemble of extremely faint flaring events, or nanoflares, are a theorized mechanism for observed large-scale heating of the solar corona (e.g., Parker 1988; Klimchuk 2006). Nanoflares would be faint and frequent enough that it would currently be impossible to detect them individually. They would occur across the entire corona, even in quiet regions with little evidence of large-scale magnetic activity. In this way, their combined effect could provide the energy necessary for coronal heating, which is not sufficiently accounted for by the energy released in observed flare populations (e.g., Hudson 1991).

It has been proposed that nanoflares originate from a reconnection process similar to that of standard flares but at a much smaller energy scale (e.g., Parker 1988). It remains unknown how frequently they might occur, whether they also accelerate particles, and the amount of energy that they could release. To refine our understanding of the emission we might 
expect from nanoflares, it is essential to investigate how flare properties change over a wide range of magnitudes. This particularly motivates the study of small microflares observed by current-generation focusing instruments, the faintest events ever observed in HXRs.

This paper provides a detailed analysis of 11 microflares observed by NuSTAR, with an emphasis on characterization of their higher-energy spectral properties and examination of the correspondence between their temporal and spatial properties and those of larger flares. To provide context, Section 2 presents an overview of the process and history of NuSTAR solar observation and also introduces the host of microflares. Sections 3 and 4 include consideration of their temporal and spatial properties in context with those of larger "standard" sized flares. Finally, Section 5 considers the spectral properties of each observed event, determining for each whether the emission is best explained by a multithermal plasma model alone or a combination of thermal and nonthermal components.

\section{NuSTAR Solar Observation}

NuSTAR is a NASA Small Explorer mission launched in 2012. It has two coaligned focusing X-ray optics designed to observe in the 3-79 $\mathrm{keV}$ band (with the range down to $2.5 \mathrm{keV}$ usable in some high-flux observations; Grefenstette et al. 2016), 18" angular resolution (FWHM), and a $12^{\prime} \times 12^{\prime}$ field of view (FOV; Harrison et al. 2013). Data from the two telescopes are identified by reference to the focal plane module (FPM) associated with each detector (FPMA, FPMB). NuSTAR is an astrophysical mission, and as such, it faces limitations when used for solar observation (Grefenstette et al. 2016). In particular, high flux rates can cause low detector livetime when observing brighter solar events, making NuSTAR primarily suitable for observation of small flares, quiescent active regions, and the quiet Sun. NuSTAR also has a pointing uncertainty of up to a few arcminutes in absolute astrometry when observing the Sun, as its forward-facing startracking camera is blinded by the solar disk (e.g., Glesener et al. 2017). To mitigate this uncertainty, NuSTAR data can be coaligned with extreme-ultraviolet (EUV) context data.

NuSTAR can experience abrupt jumps in pointing associated with changes in the combination of star-tracking camera head units (CHUs) being used to determine its orientation (Grefenstette et al. 2016). The occurrence of these shifts is well documented within NuSTAR data structures and considered in every stage of the analysis process. The CHU shifts can restrict which time intervals can be easily used for spectroscopy.

Despite these limitations, NuSTAR has completed a growing number of solar observation campaigns over the last few years, many of which have included observation of both active region microflares and quiet Sun brightenings. The magnitudes of these small events are generally compared in terms of their GOES class, a flare classification scheme based on X-ray brightness in the 1-8 $\AA$ range as observed by GOES satellites. The NuSTAR microflares studied so far have all been A-class or smaller, implying a brightness below $10^{-7}$ (A-class events) or $10^{-8}$ (sub-A-class events) watts $\mathrm{m}^{-2}$.

NuSTAR observations have allowed multiple detailed studies of sub-A-class events in active regions, as well as one paper concerned with three even smaller (GOES A0.01) quiet Sun brightenings (Glesener et al. 2017; Wright et al. 2017; Kuhar et al. 2018; Cooper et al. 2020). The spectra of events in Glesener et al. (2017), Kuhar et al. (2018), and Cooper et al. (2020) were best fit by isothermal spectral models throughout their evolution, though the Glesener et al. (2017) microflare displayed some high-energy excess over this fit during the impulsive phase. Preflare, postflare, and decay-phase spectra of the event presented in Wright et al. (2017) were also best fit by a single thermal model, but the addition of a second higher-temperature thermal model was required to account for high-energy excess during its impulsive phase.

Additionally, two papers discuss slightly larger events. One considers an A1-class microflare that is the first observed by both NuSTAR and the Interface Region Imaging Spectrograph (IRIS), making it the first event at this scale where HXR coronal emission has been compared with corresponding cooler chromospheric UV emission as seen by IRIS (Hannah et al. 2019). The other presents a GOES $A 7.7^{8}$ event that is the first NuSTAR microflare to show clear evidence of nonthermal emission (Glesener et al. 2020). This last event occurred alongside several other microflares during a 2017 August 21 NuSTAR observation and is also discussed in this paper.

Limited NuSTAR solar observing time means that full statistical A- or sub-A-class microflare studies will have to wait for the introduction of a solar-dedicated focusing HXR mission. However, it is still valuable to strive for a more systematic understanding of these uniquely faint solar brightenings than we can gain from single-event studies. This motivates the analysis of the 11 events presented here.

\subsection{Overview of Events}

On 2017 August 21, NuSTAR observed a solar active region for an orbit of around $1 \mathrm{hr}$ (NuSTAR observation IDs 20312001001 and 20312002001). This observation was granted in conjunction with the "Great American Eclipse" and ended with the eclipse of NuSTAR's FOV on the Sun by the Moon. Before the eclipse, four microflares of (backgroundsubtracted) GOES A class or below occurred in the NuSTAR FOV, all originating within the targeted active region (NOAA designation AR 12671). The evolution of emission during the single orbit is shown in the top left panel of Figure 1.

On 2018 May 29, NuSTAR observed two solar active regions over the course of five orbits, each around $1 \mathrm{hr}$ in duration. During this time, NuSTAR recorded HXR emission from seven microflares, also all A-class or below. Six of these events were initially identified by visual inspection of NuSTAR lightcurves. The last (may1917) was identified after a more rigorous method for identifying transients was applied. This set a series of conditions on the derivative of the NuSTAR lightcurve to identify flare-like local maxima based on the flarefinding algorithm used in Christe et al. (2008) for a statistical study of RHESSI microflares. All of the May events occurred during the first three orbits (NuSTAR observation IDs 80410201001, 80410201002, and 80410201003) and within the same active region (NOAA designation AR 12712). NuSTAR lightcurves for these three orbits are shown in the remaining panels of Figure 1.

NuSTAR observation of this particular region was motivated by the opportunity for co-observation with the Hi-C 2.1 sounding rocket, the flight of which occurred between two of the NuSTAR observation intervals. Other co-observing

\footnotetext{
8 In Glesener et al. (2020), the background-subtracted GOES class for this event was reported as A5.7; the difference is due to the use of reprocessed GOES data released in 2020 May for this study.
} 

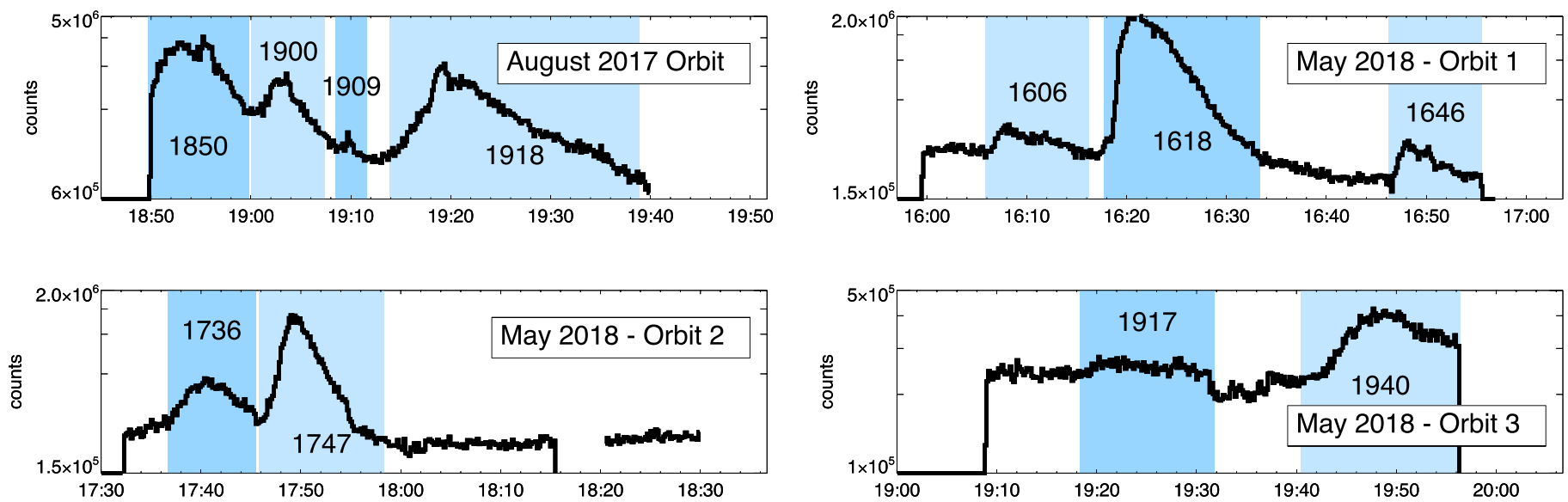

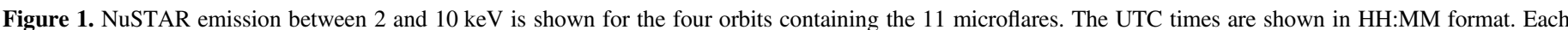

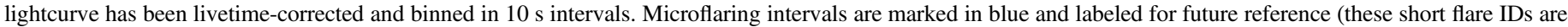

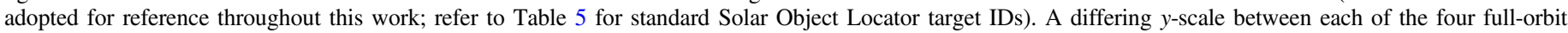

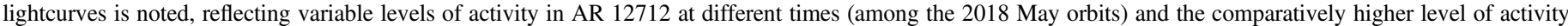
observed from AR 12671 in 2017 August.

instruments included the IRIS high-resolution UV slit spectrometer, the Hinode X-Ray Telescope (XRT) and ExtremeUltraviolet Imaging Spectrometer (EIS), and the Atmospheric Imaging Assembly (AIA) on board NASA's Solar Dynamics Observatory (SDO; Lemen et al. 2012). Detailed analysis of AR 12712 during the quiescent interval of the Hi-C 2.1 flight is presented in Warren et al. (2020). The opportunity to incorporate results from Hinode and IRIS together with the NuSTAR data set is noted as an exciting area of future investigation.

\section{Temporal Evolution}

The temporal structure of HXR emission in large flares $(>B$ class) is commonly impulsive, exhibiting a fast rise followed by a gradual fall. This is understood to imply an initial rapid release of energy to plasma heating and/or particle acceleration, followed by a lengthier decay interval as the heated plasma cools back down to temperatures below those that emit in the HXR band (e.g., Benz 2016).

The time profile of higher-energy HXR emission is generally observed to be more impulsive than that of the lower-energy emission (lower-energy HXRs or SXR emission and lower energies) and also to peak earlier in time. This is consistent with a transfer of energy from accelerated particle populations and smaller, hotter plasma volumes into heating of the surrounding chromospheric plasma, as well as with gradual cooling over time. Both impulsivity and differential peak times between energy ranges are considered part of the "standard" flare model (e.g., Benz 2016), and consistent observation of them in microflare events would support the idea that the evolution of events at this scale is controlled by processes similar to those that lead to large flares.

\subsection{Time Profile Analysis Method}

To examine these properties in the microflares considered here, four HXR energy bands were chosen within the observed NuSTAR energy range $(2-4,4-6,6-8$, and 8-10 keV). An event asymmetry index $\left(A_{\mathrm{ev}}\right)$ calculated from the rise and decay times $\left(t_{\text {rise }}, t_{\text {decay }}\right)$ was used to examine the impulsivity in all 44 cases ( 4 energy bands $\times 11$ events). This index was previously utilized to characterize the events in a RHESSI microflare study
(Christe et al. 2008), following the example of Temmer et al. (2002). It is given as

$$
A_{\mathrm{ev}}=\frac{t_{\text {decay }}-t_{\text {rise }}}{t_{\text {decay }}+t_{\text {rise }}}
$$

with a resulting value greater than zero implying an impulsive event.

Time profiles were created, including all NuSTAR emission (FPMA, FPMB summed) observed in each energy range, integrated over selected regions. For 2018 May, the regions chosen encompassed the full active region (AR 12712), which involved a relatively compact set of loops. In contrast, the 2017 August active region (AR 12671) was more structurally complex and highly elongated. In order to isolate microflarespecific temporal behavior, the regions chosen for August microflares included only the western half of the active region, the location of all four flare sites (see Figure 7 for NuSTAR microflare emission plotted over AIA active region context data).

The time profiles included livetime-corrected NuSTAR counts from several minutes before and after any flare emission was noticeable by eye, binned in $1 \mathrm{~s}$ intervals. For a few events, the microflare either began or ended outside of the period of NuSTAR observation, in which case as much of the flare time interval was included as possible.

An automated method was developed to extract flare start, peak, and end times from each time profile. A model composed of a linear combination of skewed Gaussian and linear functions (to represent flaring and background emission, respectively) was fit to each time profile using the LMFIT Python package (Newville et al. 2014). The skewed Gaussian model was chosen for its ability to flexibly fit both impulsive and nonimpulsive time profiles. The combination of the two functions requires six parameters to be fit (for the skewed Gaussian: the center, width $(\sigma)$, amplitude, and skewness $(\gamma)$; for the linear component: the slope and intercept).

Fit quality was observed to be sensitive to the choice of initial conditions, so the fitting process was repeated iteratively for an array of initial conditions for three of the fit parameters (the Gaussian center, $\sigma$, and amplitude). Optimal sets of initial conditions were found (those resulting in the best fit, with the 

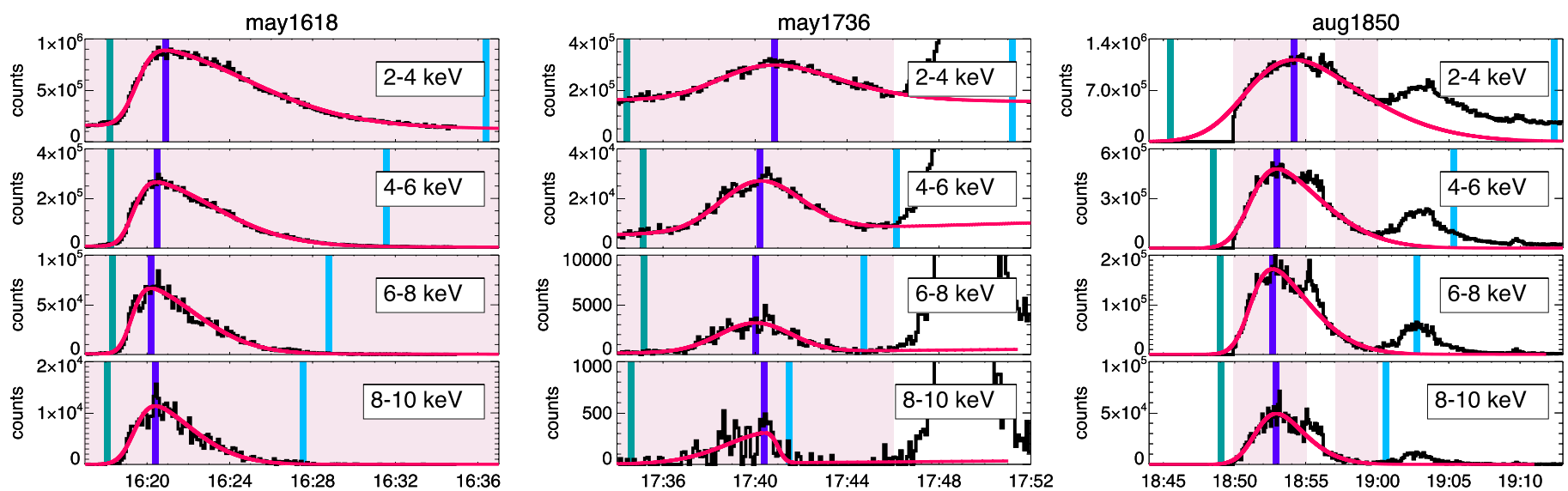

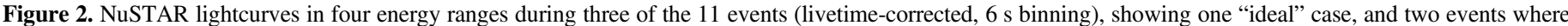

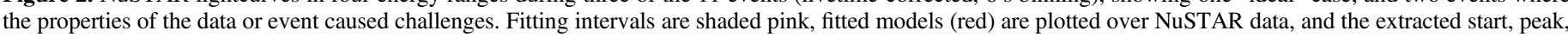

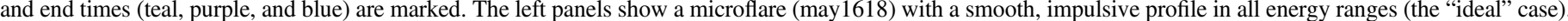

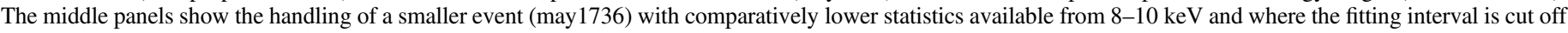

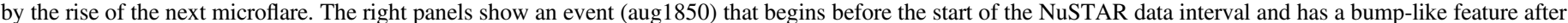
the peak that prompted further trimming of the fitting interval to achieve a reasonable result.
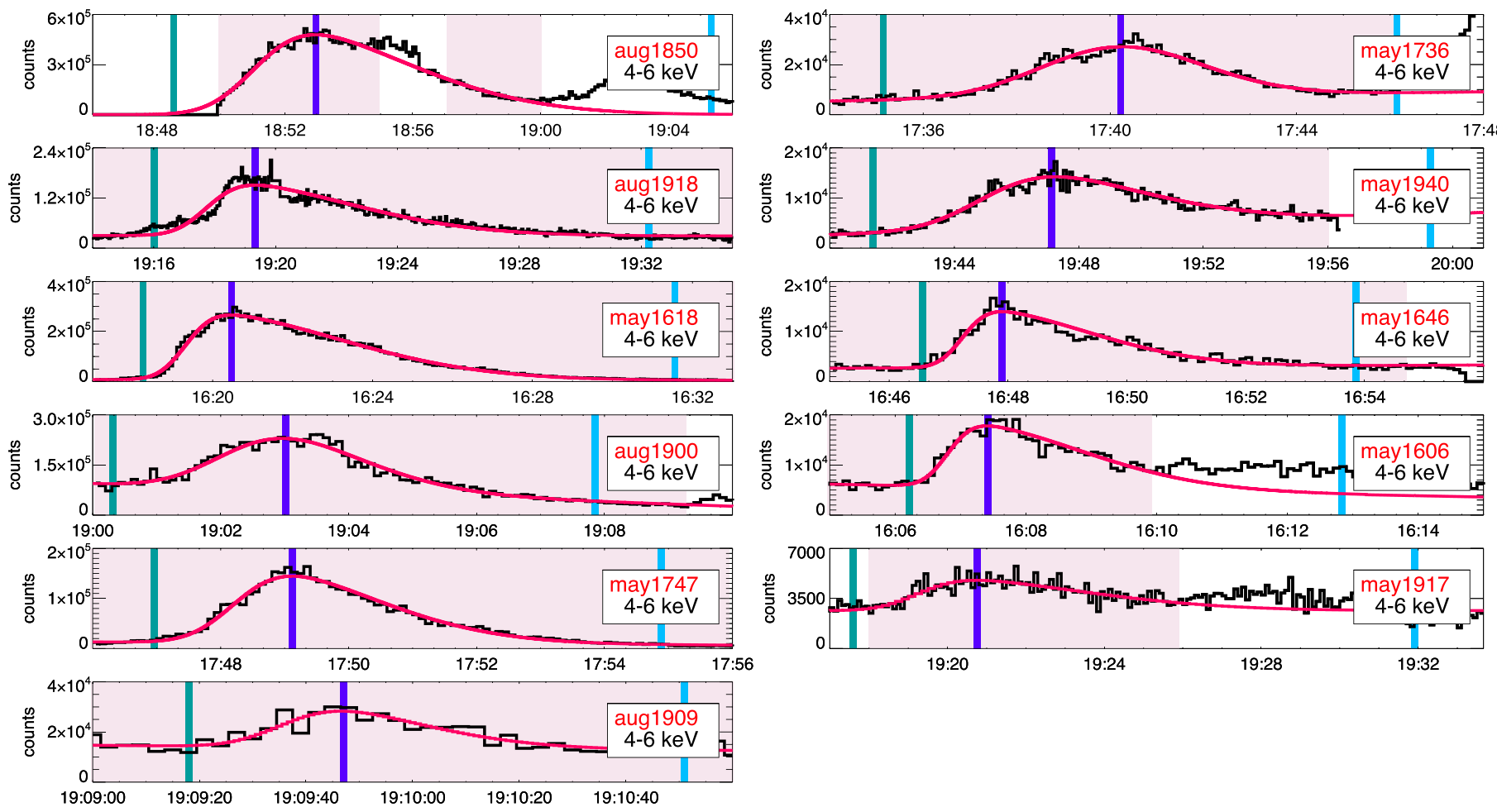

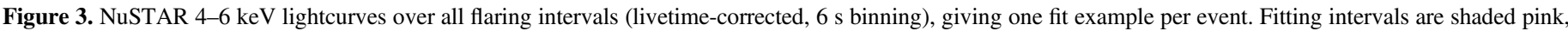

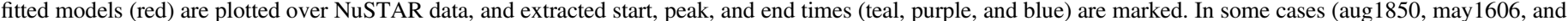

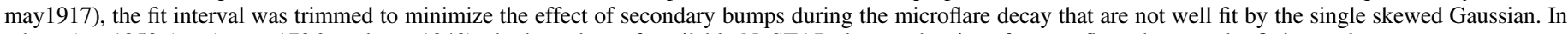
others (aug1850 (start), may1736, and may1940), the boundary of available NuSTAR data or the rise of a new flare shortens the fit interval.

goodness of fit determined via the $\chi^{2}$ value). Using these, the best-fit parameters were extracted. This was repeated for each of the 44 time profiles. Figure 2 shows the available NuSTAR data, the interval used for fitting, and the fit results in all four energy ranges for three selected events, while Figure 3 shows the 4-6 keV fit and data for every event considered.

The peak time was defined as the time of the maximum of the resulting model function. Start and end times were defined as times when the integral of the skewed Gaussian model component (with the background component removed) was equal to $0.1 \%$ and $99.9 \%$ (respectively) of its value when evaluated over the full input duration. These thresholds are arbitrary but produced reasonable start/stop times in comparison to what was apparent to the eye for each event where we had a clean observation (NuSTAR data over the full duration, with no overlapping events; see Figure 3, microflares aug1918, may1618, aug1900, may1747, aug1909, and may1646). The resulting degree of impulsivity was not strongly dependent on the exact values of the thresholds.

For 4/11 microflares (aug1850, may1646, may1736, and may1940), the full evolution of the event was not captured in the NuSTAR data; the interval was cut short by either the 
Table 1

Event Asymmetries $\left(A_{\mathrm{ev}}\right)$, Shaded to Indicate Sign of Values

\begin{tabular}{lcccc}
\hline \hline Event & $2-4 \mathrm{keV}$ & $4-6 \mathrm{keV}$ & $6-8 \mathrm{keV}$ & $8-10 \mathrm{keV}$ \\
\hline aug1850 & $0.36 \pm 0.08$ & $0.47 \pm 0.13$ & $0.47 \pm 0.14$ & $0.32 \pm 0.23$ \\
\hline aug1918 & $0.55 \pm 0.02$ & $0.59 \pm 0.02$ & $0.78 \pm 0.02$ & $0.79 \pm 0.10$ \\
\hline may1618 & $0.70 \pm 0.01$ & $0.67 \pm 0.01$ & $0.64 \pm 0.04$ & $0.51 \pm 0.10$ \\
\hline aug1900 & $0.43 \pm 0.02$ & $0.28 \pm 0.03$ & $0.35 \pm 0.04$ & $0.66 \pm 0.09$ \\
\hline may1747 & $0.48 \pm 0.02$ & $0.46 \pm 0.02$ & $0.37 \pm 0.10$ & $0.31 \pm 0.22$ \\
\hline aug1909 & $0.69 \pm 0.10$ & $0.38 \pm 0.75$ & $0.90 \pm 4.6$ & $0.54 \pm 0.63$ \\
\hline may1736 & $0.23 \pm 0.03$ & $0.07 \pm 0.07$ & $-0.02 \pm 0.17$ & $-0.68 \pm 0.06$ \\
\hline may1940 & $0.46 \pm 0.03$ & $0.36 \pm 0.03$ & $0.32 \pm 0.06$ & $0.22 \pm 0.36$ \\
\hline may1646 & $0.69 \pm 0.04$ & $0.63 \pm 0.04$ & $0.46 \pm 0.14$ & $0.70 \pm 0.29$ \\
\hline may1606 & $0.62 \pm 0.05$ & $0.64 \pm 0.03$ & $0.80 \pm 0.09$ & $0.95 \pm 0.85$ \\
\hline may1917 & $0.86 \pm 0.01$ & $0.56 \pm 0.07$ & $0.28 \pm 0.28$ & $\mathrm{X}$ \\
\hline & $\begin{array}{c}\text { Color } \\
\text { key: }\end{array}$ & $\begin{array}{c}\text { Impulsive } \\
\left(A_{\mathrm{ev}}>0\right)\end{array}$ & $\begin{array}{c}\text { Consistent } \\
\text { with Either }\end{array}$ & $\begin{array}{c}\text { Nonimpulsive } \\
\left(A_{\mathrm{ev}} \leqslant 0\right)\end{array}$ \\
\hline
\end{tabular}

Note. Here $A_{\text {ev }}$ was calculated using $t_{\text {rise }}$ and $t_{\text {decay }}$ found independently in each energy range. Due to poor statistics, the $8-10 \mathrm{keV}$ range for the faintest event (may1917) was excluded from the analysis.

boundaries of the observation or another flaring event occurring shortly after. With the use of fit results, start/end times were estimated even beyond the available NuSTAR data in these cases. Additionally, three events (may1850, may1606, and may1917) contained bumps in the decay interval that distorted the fit results, pushing the end times well beyond a value that seemed physical. For these events, the interval used for fitting was manually trimmed to avoid including these features.

Uncertainty in peak times was dependent on LMFIT output $1 \sigma$ standard errors in both the center and $\gamma$ of the distribution, while uncertainties in start and stop times were additionally dependent on the error in $\sigma$. To extract uncertainties, fits were iteratively rerun for each time profile wherein the center, $\gamma$, and $\sigma$ were randomly assigned to values within their output error range each time and then held fixed while the other three parameters remained free. Peak, start, and end time uncertainties were taken as the standard deviation of their resulting values.

\subsection{Peak Times and Impulsivity}

Table 1 shows the event asymmetry index, $A_{\mathrm{ev}}$, for each microflare in each of the four energy ranges. Events are arranged from brightest (top) to faintest (bottom) considering the maximum NuSTAR count rate during each (livetimecorrected and background-subtracted). The majority (36/43; dark green) of the time profiles are confirmed to be impulsive, and six more (light green) are consistent with either an impulsive or a nonimpulsive evolution.

One event (aug1909) was consistent with both impulsive and nonimpulsive profiles in $3 / 4$ energy ranges. This was the shortest-duration microflare $(<2$ minutes). While start, peak, and end time uncertainties in this event were not notably larger than those found for others, they were larger in proportion to the flare duration, leading to large uncertainty ranges in $A_{\mathrm{ev}}$ in the higher energies. The lack of confirmed impulsivity in these

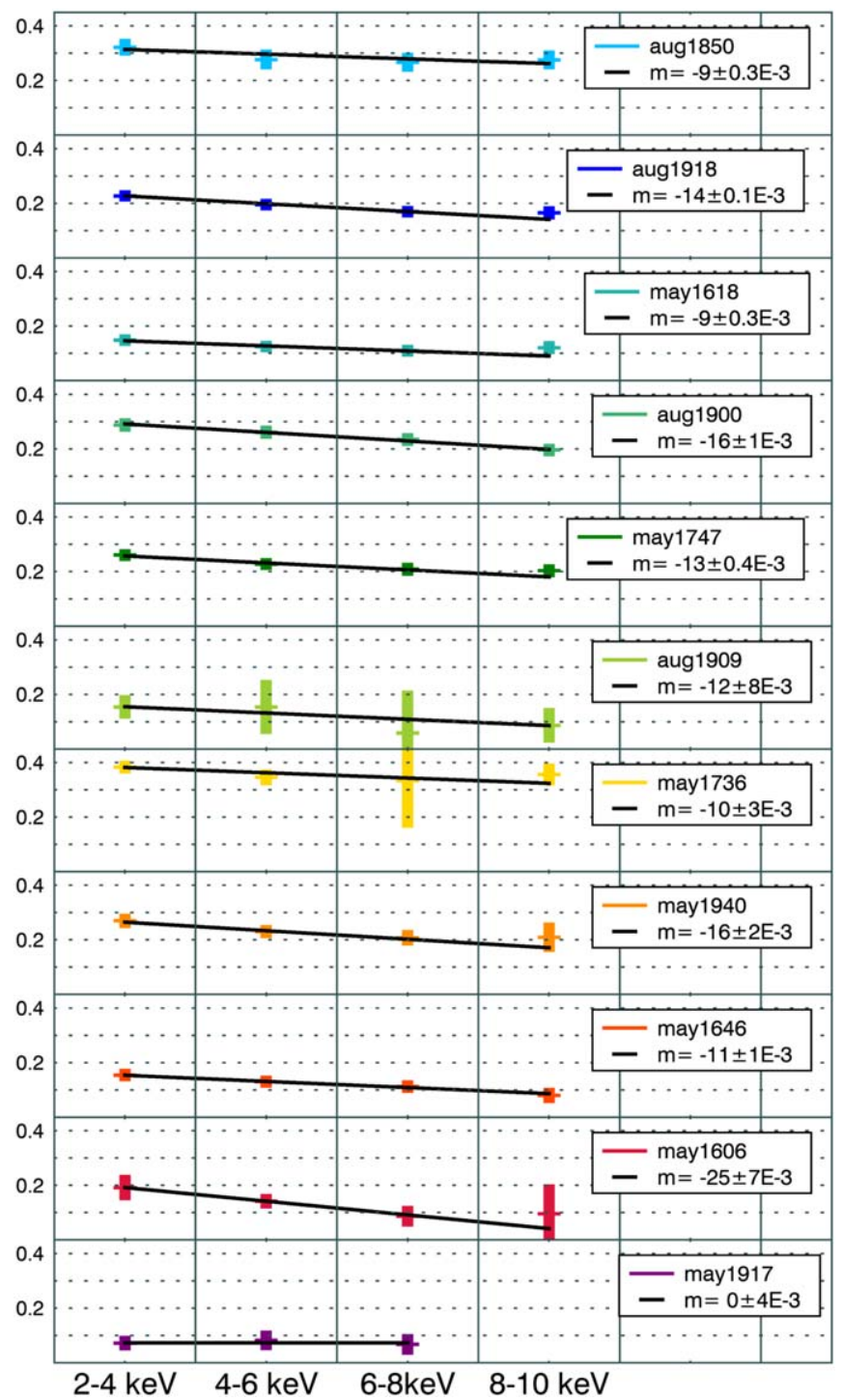

Figure 4. Microflare peak times are shown normalized over the $2-4 \mathrm{keV}$ event duration such that a value of zero would imply that the microflare peaks the moment it begins, while a value of 0.5 would imply a peak halfway through the duration. Peak times are shown in all four energy ranges, with error bars showing uncertainties for each. A linear fit is included for each event, and resulting slopes $(\mathrm{m})$ are reported with $1 \sigma$ uncertainties. The data are consistent with a negative slope only in 10/11 cases (a slope of zero was found for the faintest event, may1917, which had sufficient counts to be well fit in only three energy ranges). This shows a trend toward earlier peak times in the higher energy ranges. Events are arranged from brightest (top) to faintest (bottom) by the maximum NuSTAR count rate (livetime-corrected and backgroundsubtracted) during each interval.

time profiles therefore reflects a limitation of the available statistics.

The reported values of $A_{\mathrm{ev}}$ represent the location of the time profile peak within the event duration in each energy range. It is also interesting to compare time profile peaks in each energy range to the full event duration (defined as the interval from the earliest start to the latest end found in any of the energy ranges for each event; in all 11 microflares, this was equal to the $2-4 \mathrm{keV}$ event duration). The peak times of emission in all energy ranges for each microflare are shown in Figure 4. In order to visually compare between energy ranges for all microflares simultaneously, the peak times were normalized over each $2-4 \mathrm{keV}$ event duration. Linear trends in peak time 
across the four energy ranges were calculated for each microflare. Ten of the 11 resulting trend lines had negative slopes, confirming the observation of the large flare property of earlier peak times in higher-energy emission.

\subsection{Hardness Ratios}

The differential flux spectrum of thermal bremsstrahlung from a volume of plasma is dependent on the electron and ion densities $\left(n_{\mathrm{e}}, n_{\mathrm{i}}\right)$, as well as the temperature $(T)$ of the plasma. It is given as a function of emitted energy, $\epsilon$, as

$$
F(\epsilon) \approx 8.1 \times 10^{-39} \int_{V} \frac{\exp \left(\frac{-\epsilon}{k_{B} T}\right)}{T^{\frac{1}{2}}} n_{i} n_{e} d V
$$

$\left(\mathrm{keV} \mathrm{s}^{-1} \mathrm{~cm}^{-2} \mathrm{keV}^{-1}\right)$, where factors on the order of 1 have been neglected, and the integral is taken over the volume of emitting plasma (Aschwanden 2005). The ratio of this flux at two different energies can be shown to be a monotonically increasing function of $T$.

With sufficient knowledge of instrument response, this relationship can be used to determine the evolution of flare temperature in absolute terms (as was done for a large population of GOES flares in Ryan et al. 2012). NuSTAR's energy resolution allows for flare temperatures to be more accurately extracted from spectroscopy (see Section 5). However, the need to include enough counts to make spectral fitting meaningful limits the temporal resolution possible when examining the evolution of flare plasma parameters over the course of an event. Hardness ratios (ratios between counts in higher and lower NuSTAR energy ranges) do not have this limitation.

Two different hardness ratios were examined in these events: $R_{4 / 2}$ (ratio of 4-6 and 2-4 keV emission) and $R_{8 / 4}$ (ratio of 8-10 and $4-6 \mathrm{keV}$ emission). Figure 5 shows both ratios as a function of time during two example events, with normalized NuSTAR emission in all four energy ranges included for context. The hardness ratios are normalized over the flaring interval for visual convenience in comparing between $R_{4 / 2}$ and $R_{8 / 4}$.

These events are representative of the population of microflares, all of which showed ratios with structure similar to that of the regular NuSTAR time profiles, peaking either simultaneously or earlier in time. The exceptions to this were smaller events, where limited statistics in the $8-10 \mathrm{keV}$ energy range challenged the interpretation of $R_{8 / 4}$.

Table 2 gives peak times in each of the hardness ratios for each microflare, with the events again arranged by magnitude of peak NuSTAR counts (livetime-corrected, backgroundsubtracted). The ratio peak times are reported as fractions of the full 2-4 keV event duration in each case. Uncertainties were found by applying a range of different smoothing intervals to each ratio curve before taking the maximum and using the standard deviation of the resulting peak times as the reported uncertainty. The uncertainty associated with the choice of smoothing interval was seen to dwarf that due to the inherent statistical uncertainty of the NuSTAR data. For both ratios in all 11 microflares, the peak occurs in the first half of the event. The mean values of the peaks (times of maximum microflare temperature assuming an isothermal emitting plasma) are $0.176 \pm 0.034\left(R_{4 / 2}\right)$ and $0.156 \pm 0.043\left(R_{8 / 4}\right)$ when averaged over all events except aug1850.
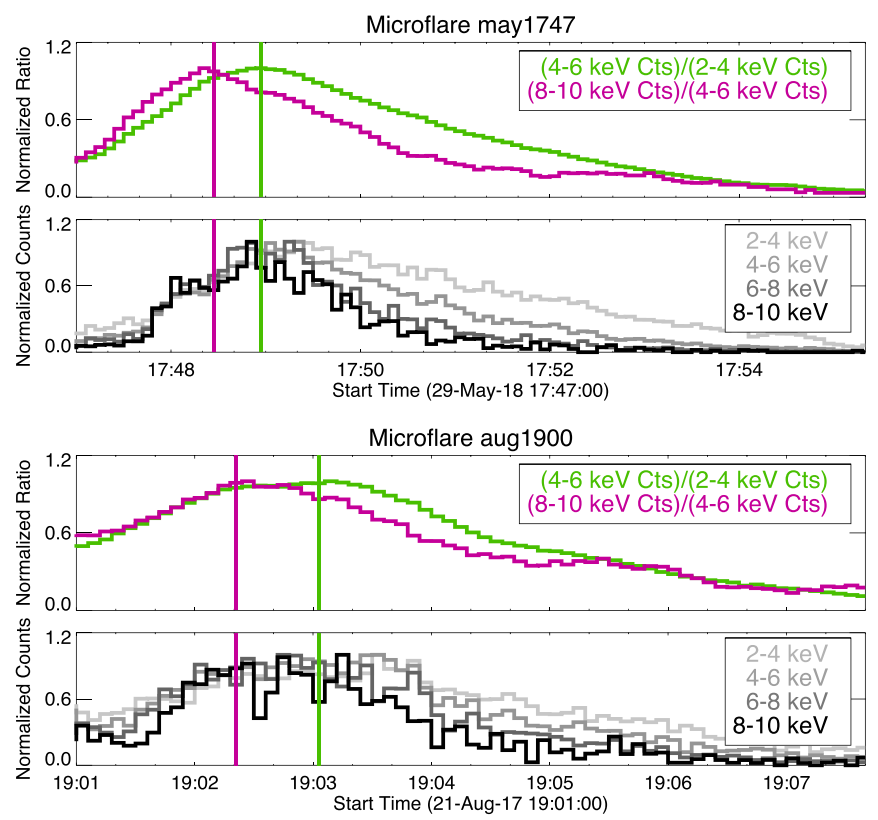

Figure 5. The time evolution of the ratios between two sets of NuSTAR energy ranges is shown for two example events, a proxy for temperature. Ratio curves have been smoothed over a $10 \mathrm{~s}$ interval, and their peak times are marked with color-coded vertical lines and extended down for comparison with normalized NuSTAR emission in four energy ranges. In the top event (may1747), the two ratios peak before the peaks in emission in any of the NuSTAR channels, while they straddle the NuSTAR peaks in the bottom event (aug1900).

Table 2

Hardness Ratio Peak Times (Fraction of Flare Duration)

\begin{tabular}{lcc}
\hline \hline Event & $R_{4 / 2}$ & $R_{8 / 4}$ \\
\hline aug1850 & $0.264 \pm 0.006$ & $0.277 \pm 0.008$ \\
\hline aug1918 & $0.183 \pm 0.015$ & $0.178 \pm 0.020$ \\
\hline may1618 & $0.096 \pm 0.025$ & $0.111 \pm 0.036$ \\
\hline aug1900 & $0.271 \pm 0.026$ & $0.198 \pm 0.023$ \\
\hline may1747 & $0.203 \pm 0.019$ & $0.165 \pm 0.022$ \\
\hline aug1909 & $0.038 \pm 0.139$ & $0.125 \pm 0.097$ \\
\hline may1736 & $0.339 \pm 0.019$ & $0.296 \pm 0.044$ \\
\hline may1940 & $0.226 \pm 0.012$ & $0.184 \pm 0.039$ \\
\hline may1646 & $0.148 \pm 0.036$ & $0.102 \pm 0.041$ \\
\hline may1606 & $0.173 \pm 0.028$ & $0.161 \pm 0.038$ \\
\hline may1917 & $0.081 \pm 0.016$ & $0.043 \pm 0.071$ \\
\hline
\end{tabular}

Note. The significant nonthermal contribution to emission in aug1850 (see Section 5) complicates the interpretation of the ratio peak as a temperature peak in this case.

\subsection{Neupert Effect}

The Neupert effect describes the tendency for flaring HXR or microwave emission to show a correlation with the derivative of the lightcurve of emission in lower energy ranges, as noted in Neupert (1968). Observation of this property is interpreted to support the idea that plasma heating resulting in EUV and SXR emission is caused by the deposition of energy by beams of nonthermal accelerated electrons, which are in turn the source of emission in the HXR band (Dennis \& Zarro 1993). 
In order to look for evidence of this, cotemporal lower-energy emission was examined in conjunction with higher-energy (6-8 and 8-10 keV) NuSTAR time profiles. The SXR emission was taken from the GOES $1-8 \AA$ passband and the EUV from the SDO/AIA $94 \AA$ channel, the latter spatially integrated over the relevant active regions. This meant that, in total, 44 pairs of lightcurves were examined ( 2 NuSTAR energy ranges $\times 11$ microflares $\times 2$ lower-energy instruments).

The AIA and GOES time profiles were smoothed over 2 minute boxcar intervals before their derivatives were taken, with the aim of highlighting longer-term temporal structure over background fluctuations. This was modified in the case of aug 1909, where the GOES and AIA emission were smoothed over 1 minute instead due to the $<2$ minute event duration. Intervals were selected over which to compare NuSTAR emission with the GOES or AIA derivatives, including only the times where the GOES or AIA derivatives were nonnegative. NuSTAR data were binned to match the cadence of the lowerenergy instruments, and emission in each of the instruments was normalized.

Cross-correlation between each pair of lightcurves was computed using the C_CORRELATE function in IDL, reporting the maximum correlation coefficient found and its associated lag. Figure 6 shows the best-correlated result (from either the 6-8 or 8-10 keV comparison) for both GOES and AIA for two events.

In 18/22 AIA and 17/22 GOES comparisons, the best correlation between NuSTAR emission and the lower-energy derivative was found after a positive shift of the derivative in time (positive lag), implying that the lower-energy derivative peaks earlier in time than the NuSTAR emission. An example of this can be seen in the top panel of Figure 6 .

If an increase in the amount of plasma emitting in a given SXR or EUV energy range were the result of heating by a nonthermal particle population, the derivative would be expected to peak at the same time as (or later than) the nonthermal emission. In contrast, events best correlated with a positive lag are explainable by entirely thermal emission in both the HXR and SXR/EUV ranges and are not consistent with the Neupert effect. This appears to be the dominant behavior among this population.

Considering the events not best correlated when a positive lag is applied, the majority were cases in which the correlation between NuSTAR and the lower-energy derivative was weak or seemed unphysical. Some of the smallest events were faint enough to be difficult to discern in the GOES light curves, but even some that were visible did not display a strong correlation. This is likely indicative of a more complex physical situation than is assumed by either the Neupert effect or the simple thermal scenario described above.

Microflare aug1850, which was confirmed to involve significant nonthermal emission in Glesener et al. (2020), is the only event to show behavior consistent with the Neupert effect with the use of this method. Specifically, the AIA derivative is best correlated with both the NuSTAR 6-8 and $8-10 \mathrm{keV}$ time profiles when no lag is applied at all (see bottom panel of Figure 6). The GOES derivative, while requiring a small positive shift to achieve the best mathematical correlation, does also qualitatively appear well correlated with the rise of the NuSTAR emission in both energy ranges without being shifted at all (6-8 keV correlation coefficients: 0.399 (no shift),
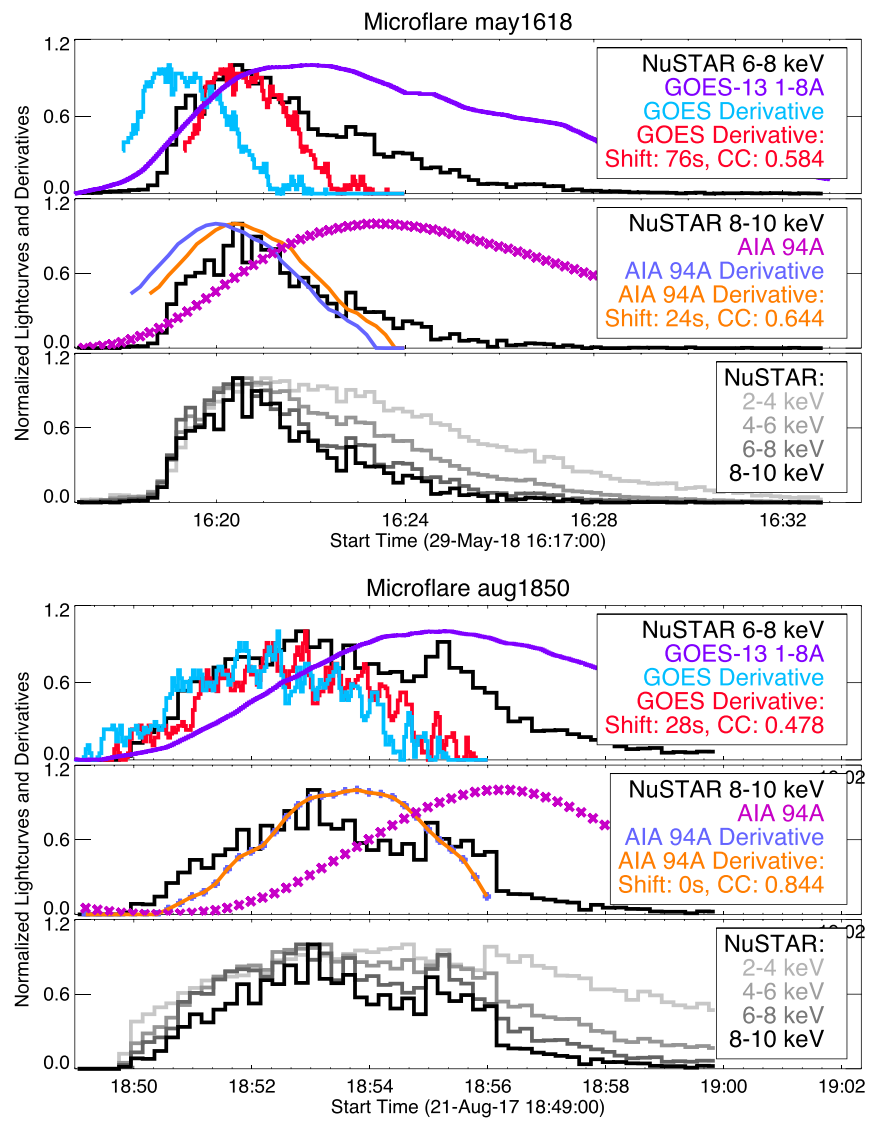

Figure 6. Correlation results between NuSTAR and the derivatives of lowerenergy emission for two events. For each event, three plots are shown. The top plot shows GOES derivatives over the cross-correlation interval, with full flare duration NuSTAR and raw GOES lightcurves for context. The middle plot shows the same, comparing NuSTAR and AIA. In the bottom plot, normalized NuSTAR emission is shown in all four energy ranges over the flare duration. Legends for the GOES and AIA lightcurves include the correlation coefficient (CC) and associated shift. In the top panel, the may1618 time profiles are best correlated with AIA $94 \AA$ and GOES 1-8 A derivatives when they are shifted forward in time (not consistent with the Neupert effect). The bottom panel shows aug1850, an event confirmed to contain nonthermal emission. The $8-10 \mathrm{keV}$ time profile is best correlated with the AIA $94 \AA$ derivative with no shift, and the rise in $6-8 \mathrm{keV}$ is well correlated with the GOES 1-8 $\AA$ derivative even before a shift is applied to maximize the mathematical correlation (consistent with the Neupert effect).

0.478 (28 s shift); $8-10 \mathrm{keV}$ correlation coefficients: 0.357 (no shift), 0.457 (58 s shift)).

\section{Spatial Properties}

NuSTAR's imaging capabilities allow for comparison between the spatial distribution of observed HXR emission and that of EUV emission observed by SDO/AIA. NuSTAR's $18^{\prime \prime}$ angular resolution (FWHM) means that structure on the scale of larger active region loops can be resolved, though much of the finer loop spatial structure visible in AIA is not. As a first step in investigating the spatial properties of the observed emission, NuSTAR's pointing stability was examined over each flaring interval. In 10/11 microflares, the CHU combination and pointing were stable over the entire flare (may1606, may1736, may1917, may1940, aug1900, and aug1909) or over the rise and peak times (may1646, may1618, may1747, and aug1918). For these events, data from the dominant (or rise/ peak) CHU combination were used to make images, reducing event duration in some cases (the same intervals were later used 

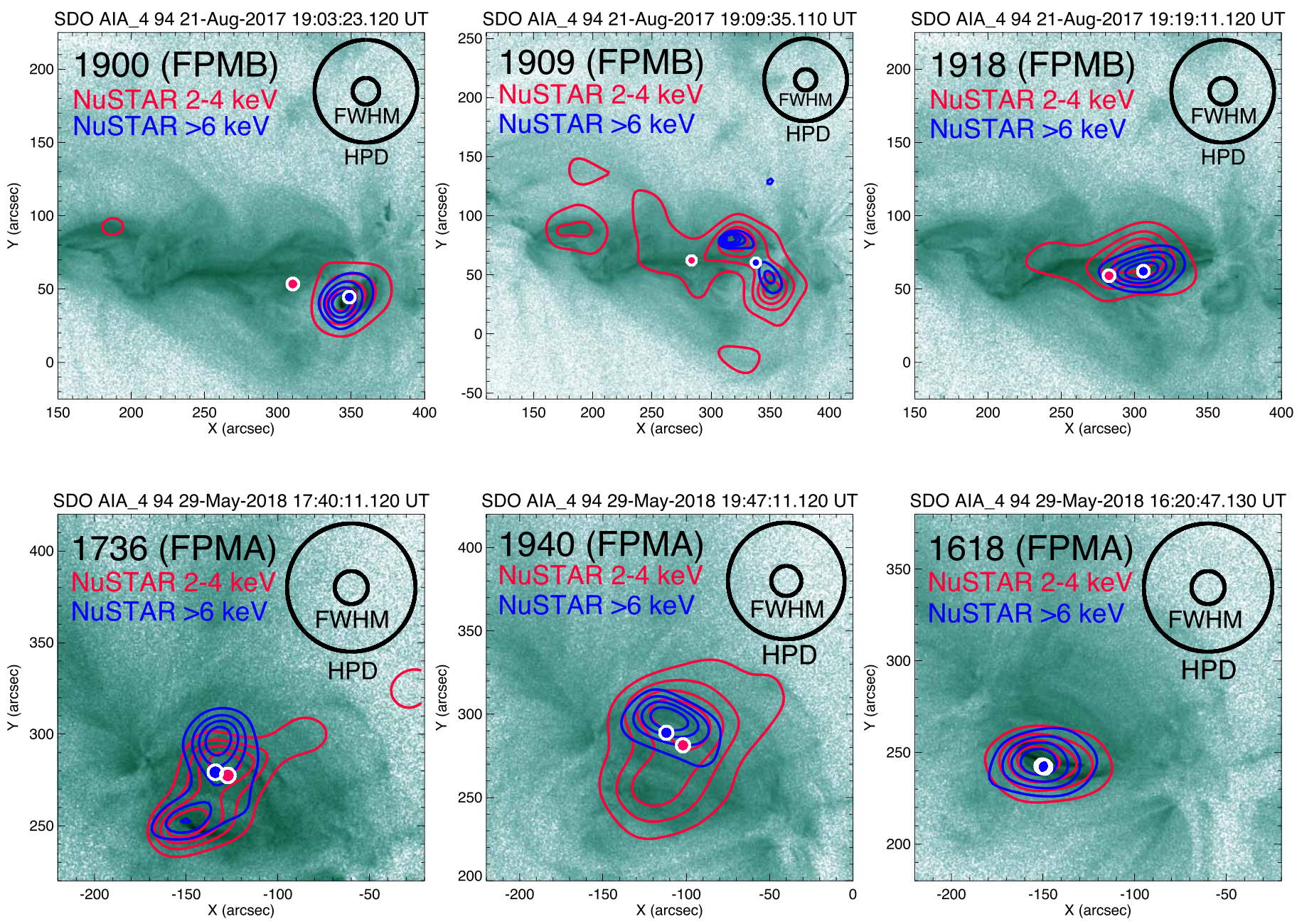

Figure 7. NuSTAR contours $(20 \%, 40 \%, 60 \%$, and $80 \%$ of counts) are shown over AIA $94 \AA$ context images during six of the 11 microflares. NuSTAR $2-4$ and $>6 \mathrm{keV}$ emission during each event has been integrated over stable pointing intervals and deconvolved over flare- and energy range-specific numbers $(i)$ of iterations. Emission centroids in each energy range are also marked. Circles with diameters equal to the NuSTAR half power diameter ( $\lesssim 70^{\prime \prime}$ in this energy range) and FWHM $\left(18^{\prime \prime}\right)$ are shown for visual reference (Madsen et al. 2015). The top panels show three of the 2017 August microflares, each with unique morphology involving different parts of the complex, multiloop structure of AR 12671 (see Glesener et al. 2020 for images of microflare aug1850). No preflare background has been removed, as no times during the NuSTAR observation of this region could be considered quiescent. The same number of iterations was used for deconvolution of each (2-4 keV: $i=200 ;>6 \mathrm{keV}: i=100$ ). In the bottom panels, in contrast, emission in all 2018 May events is dominated by the contributions of two similarly shaped structures (an "upper" and "lower" set of loops). The bottom left panel shows microflare may1736 (no preflare background has been removed in this case; $2-4 \mathrm{keV}: i=100$; $>6 \mathrm{keV}: i=50$ ). This event involved significant NuSTAR emission from both the upper and lower loop structures. The middle panel shows may1940, an event primarily involving the upper loop structure $(2-4 \mathrm{keV}: i=100$; $>6 \mathrm{keV}: i=50$ ), while the right panel (may1618) shows one dominated by the lower (2-4 keV: $i=200 ;>6 \mathrm{keV}: i=100$ ). The brightest of the 2018 May microflares was may1618, and it has had its background emission removed. No significant energydependent difference in the NuSTAR centroid was found for this event.

for spectroscopy in Section 5). For aug1850, multiple CHU changes occurred during the rise/peak, so the CHU with the largest effective exposure was chosen for imaging.

Depending on the location of the detector chip gap in relation to an observed source, one FPM may be more ideal for imaging in any given observation; FPMA was better oriented during the 2018 May observation, while FPMB was better during all 2017 August events. To make images, NuSTAR emission from one FPM was integrated in time over the CHUstable intervals for each microflare. NuSTAR's point-spread function was then deconvolved over an event- and energy range-specific number of iterations using the IDL procedure max_likelihood.pro.

Because NuSTAR is sensitive at temperatures similar to those that most strongly produce the Fe XVIII line (peak formation temperature of $\log (T) \approx 6.9$; Del Zanna et al. 2015), AIA Fe XVIII images can be used to approximate the most likely true center of NuSTAR emission, reducing the instrument's inherent pointing uncertainty during solar observation. The AIA Fe XVIII images were produced using an established linear combination of three channels $(94,171$, and $211 \AA$ ) to isolate Fe XVIII emission (Del Zanna 2013). Differenced Fe XVIII images were then created (peak time in NuSTAR $2-4 \mathrm{keV}$ minus a preflare time).

Finally, for each flare, the deconvolved NuSTAR contours were manually coaligned to the differenced Fe XVIII images. Figure 7 shows NuSTAR emission from six microflares as contours over AIA $94 \AA$ context images. The number of deconvolution iterations used for each energy range in each event is given in the image caption.

\subsection{Spatial Complexity}

Differences in the centroid of flare time emission in different HXR energy ranges could provide evidence of a plasma 
temperature gradient across the flare site. Alternatively, such differences could highlight spatially distinct thermal and nonthermal sources, such as the common scenario of nonthermal loop footpoint sources in large flares observed in conjunction with thermal emission from flare loops (e.g., Benz 2016). To determine whether an event displays spatial complexity, background active region emission must first be subtracted from the flare time images. This ensures the isolation of complexity within the microflare itself, rather than just characterization of a spatial difference between flare emission and (generally lower-energy) emission from the surrounding active region.

Suitable quiet times for background subtraction were found for only two events (16:44-16:45 UT for may1618, shown in Figure 7; 18:12-18:15 UT for may1747), as essentially no quiet times occurred during the 2017 August observation (see Figure 1), and the remaining 2018 May microflares were faint enough that background subtraction resulted in poor statistics and significant nonphysical distortion. After background subtraction, the NuSTAR emission centroid was computed in the 2-4, 4-6, and $>6 \mathrm{keV}$ energy ranges for each FPM, considering all pixels with values above $15 \%$ of the maximum pixel value in each raw (not deconvolved) image. Differences in the centroid between the two FPMs in the full NuSTAR energy range (all energies $>2 \mathrm{keV}$ ) were used as an estimate of uncertainty in the centroid measurements. Neither of the events displayed a difference between emission centroids in different NuSTAR energy ranges larger than the estimated uncertainty. This is consistent with what is observed in AIA Fe XVIII, where both of these events showed dominant emission from just one feature.

\section{Spectroscopy}

Spectroscopy of the NuSTAR microflares was performed using the XSPEC spectral fitting software (Arnaud 1996). Data from NuSTAR's two telescopes and their corresponding FPMs (FPMA, FPMB) were fit simultaneously using the Cash statistic (CSTAT) as a fit statistic, which better handles lowcount data (Cash 1979).

The same stable, single CHU combination time intervals described in Section 4 were used for spectroscopy in 10/11 microflares. As previously noted, NuSTAR pointing was reconstructed using several different $\mathrm{CHU}$ combinations during event aug1850. However, large pointing shifts were not associated with the CHU changes during the first 3 minutes (rise/peak) of the microflare. Because of this, aug1850 spectroscopy was performed using data from all component CHU combinations over that interval.

Though the NuSTAR energy range is typically cited as $3-79 \mathrm{keV}$, the instrument is capable of observation down to $2 \mathrm{keV}$. However, differing pixel thresholds complicate the response at the very low end of the energy range, and spectroscopy below $2.5 \mathrm{keV}$ is not recommended (Grefenstette et al. 2016). As a conservative approach, a lower-energy bound of $3 \mathrm{keV}$ was employed for spectroscopy in this analysis. The upper bound of the fit energy range was set to be $10 \mathrm{keV}$ (for fainter events with little to no emission above that energy) or $12 \mathrm{keV}$ for the brightest events (aug1850, aug1900, aug1918, may1618, and may1747). The NuSTAR spectra were binned to have a minimum of 10 counts in each energy bin.

For each flaring interval, the effects of pileup were estimated by examining the incidence of multiple-pixel events with geometries that cannot be explained by sharing of charge from one photon between adjacent pixels (see Appendix $\mathrm{C}$ of Grefenstette et al. 2016). Pileup was found to be negligible in all events. Additionally, it is noted that when a pileup correction was performed for the brightest microflare considered here (aug 1850) in an earlier study (Glesener et al. 2020), it resulted in changes to spectral parameters small enough to be consistent within their uncertainties (Glesener, personal communication). Because of this, no pileup correction was performed for the microflares examined here. The data used for spectroscopy were filtered to consider single-pixel events only.

While the events considered in this study are small in magnitude compared to the population of flares observed by RHESSI and other previous solar observatories, they also include the brightest flares yet observed with NuSTAR under optimal observing conditions. Because of this, analysis of these events led to the first identification of variations in the NuSTAR gain in the extremely low livetime $(<1 \%)$ regime.

This phenomenon is described in detail in the Appendix, the conclusion of which is a simple correction to the slope of the linear gain, as well as a set of criteria for determining whether such a correction is likely to be necessary for a given event. Consideration of such corrections will be standard practice for future NuSTAR microflare studies. Here gain corrections were performed for aug1850, aug1900, aug1918, may1618, may1736, and may 1747 with the percent shift in gain slope recorded in Table 3. In most cases, the gain slope parameter is tied between the two FPMs, with the uncertainty in the resulting value defined as the difference between FPMA and FPMB gain slope values when the fit is rerun with the FPMA and FPMB gain slopes untied. The orientation of the detector chip gap in FPMA over a portion of the flare site during aug 1850 led to significant differences in flux between the two FPMs; because of this, the FPMA and FPMB gain slope corrections were determined independently for that event.

Initial spectroscopy used XSPEC's isothermal vapec model, which allows for user-specified abundances (taken from Feldman 1992, as is standard practice for NuSTAR solar spectroscopy; e.g., Wright et al. 2017). High-energy excess was seen over the single vapec model in all 11 events. The origin of this excess was hypothesized to be either emission from smaller volumes of higher-temperature plasma likely produced at or near the reconnection site or nonthermal emission from flare-accelerated electrons. To investigate these possibilities, a second isothermal model was added (vapec+vapec), and a separate fit was also performed using an isothermal model in conjunction with a nonthermal broken power law (vapec +bknpwr). For the bknpwr model component, the spectral index below the break energy was fixed to 2 for all events, as it was expected that the thermal component would dominate at lower energies.

\subsection{Spectral Results}

The majority $(8 / 11)$ of the microflares were best fit by the vapec+vapec model, as determined by the use of the CSTAT, as well as manual inspection of residuals. This included all 2018 May microflares and aug1909. Example spectra from one of these clearly thermal microflares are shown in Figure 8, displaying all three potential models. The thermal parameters found for these events are reported in Table 3, which also reports the estimated thermal energy present in each (the energy values reported are the sum of the thermal 
Table 3

Fit Thermal Parameters (vapec+vapec)

\begin{tabular}{|c|c|c|c|c|c|c|c|}
\hline Event & $\begin{array}{c}\text { VAPEC1 } \\
T(\mathrm{MK}) \\
\mathrm{EM}\left(\mathrm{cm}^{-3}\right)\end{array}$ & $\begin{array}{c}\text { VAPEC } 2 \\
T(\mathrm{MK}) \\
\mathrm{EM}\left(\mathrm{cm}^{-3}\right)\end{array}$ & Gain Shift & $\begin{array}{l}\text { Loop Density } \\
\quad\left(\mathrm{cm}^{-3}\right)\end{array}$ & $\begin{array}{c}\text { Energy } \\
\text { Thermal (erg) }\end{array}$ & $\begin{array}{l}\text { GOES } \\
\text { Calc. }\end{array}$ & $\begin{array}{c}\text { GOES } \\
\text { Obs. }\end{array}$ \\
\hline aug 1850 & $\begin{array}{c}10.3_{-0.2}^{+0.2} \\
5.6_{-0.3}^{+0.3} \times 10^{45}\end{array}$ & & $\begin{array}{l}0.972 \pm 0.007(\mathrm{~A}) \\
0.979 \pm 0.007(\mathrm{~B})\end{array}$ & $4.8_{-1}^{+0.6} \times 10^{9}$ & $5.0_{-0.7}^{+1.2} \times 10^{27}$ & & $\bar{A} 7.7$ \\
\hline aug 1918 & $\begin{array}{c}4.1_{-0.1}^{+0.1} \\
2.8_{-0.1}^{+0.1} \times 10^{47}\end{array}$ & $\begin{array}{c}10.0_{-0.1}^{+0.02} \\
4.4_{-0.1}^{+0.2} \times 10^{45}\end{array}$ & $0.984 \pm 0.003$ & $5.2_{-1}^{+1} \times 10^{9}$ & $9.1_{-2}^{+3} \times 10^{28}$ & $\mathrm{~A} 4.7_{-0.7}^{+0.1}$ & A 4.5 \\
\hline may 1618 & $\begin{array}{c}4.1_{-0.1}^{+0.2} \\
1.4_{-0.4}^{+0.6} \times 10^{47}\end{array}$ & $\begin{array}{c}10.0_{-0.03}^{+0.03} \\
4.6_{-0.2}^{+0.1} \times 10^{45}\end{array}$ & $0.977 \pm 0.002$ & $5.6_{-2}^{+2} \times 10^{9}$ & $4.3_{-1}^{+4} \times 10^{28}$ & A $3.0_{-0.9}^{+1.5}$ & A 8.0 \\
\hline aug 1900 & $\begin{array}{c}4.3_{-0.2}^{+0.3} \\
1.5_{-0.4}^{+0.2} \times 10^{47}\end{array}$ & $\begin{array}{c}10.0_{-0.2}^{+0.04} \\
3.9_{-0.1}^{+0.6} \times 10^{45}\end{array}$ & $0.991 \pm 0.001$ & $9.3_{-2}^{+2} \times 10^{9}$ & $2.9_{-0.4}^{+0.9} \times 10^{28}$ & $\mathrm{~A} 3.1_{-1.1}^{+1.4}$ & A 3.9 \\
\hline may 1747 & $\begin{array}{c}4.7_{-0.3}^{+0.05} \\
5.0_{-0.7}^{+0.1} \times 10^{46}\end{array}$ & $\begin{array}{c}10.1_{-0.03}^{+0.03} \\
1.2_{-0.03}^{+0.1} \times 10^{45}\end{array}$ & $0.984 \pm 0.01$ & $4.6_{-1}^{+1} \times 10^{9}$ & $2.1_{-0.5}^{+0.5} \times 10^{28}$ & $\mathrm{~A} 1.5_{-0.01}^{+0.2}$ & A4.3 \\
\hline aug 1909 & $\begin{array}{c}4.0_{-0.6}^{+0.1} \\
2.5_{-0.6}^{+2.9} \times 10^{47}\end{array}$ & $\begin{array}{c}8.2_{-0.1}^{+0.3} \\
2.6_{-0.9}^{+0.5} \times 10^{45}\end{array}$ & None & $1.8_{-0.3}^{+1} \times 10^{10}$ & $2.2_{-0.7}^{+1} \times 10^{28}$ & $\mathrm{~A} 3.8_{-2.3}^{+4.5}$ & $<\mathrm{A} 1$ \\
\hline may 1736 & $\begin{array}{c}4.2_{-0.03}^{+0.1} \\
6.9_{-1.3}^{+0.8} \times 10^{46}\end{array}$ & $\begin{array}{c}9.9_{-1.1}^{+0.3} \\
2.8_{-0.08}^{+1.8} \times 10^{44}\end{array}$ & $0.984 \pm 0.003$ & $4.9_{-2}^{+3} \times 10^{9}$ & $2.5_{-1}^{+1} \times 10^{28}$ & $\mathrm{~A} 1.2_{-0.3}^{+0.2}$ & A2.8 \\
\hline may 1940 & $\begin{array}{c}4.0_{-0.4}^{+0.05} \\
9.4_{-0.8}^{+6.1} \times 10^{46} \\
\end{array}$ & $\begin{array}{c}7.9_{-0.8}^{+0.06} \\
6.0_{-0.8}^{+4.0} \times 10^{44} \\
\end{array}$ & None & $5.5_{-0.5}^{+2} \times 10^{9}$ & $2.8_{-0.5}^{+1} \times 10^{28}$ & $\mathrm{~A} 1.5_{-0.6}^{+1.0}$ & A1.8 \\
\hline may 1646 & $\begin{array}{c}3.4_{-0.07}^{+0.1} \\
2.1_{-0.5}^{+0.5} \times 10^{47}\end{array}$ & $\begin{array}{c}8.0_{-0.4}^{+0.09} \\
5.0_{-0.6}^{+1.4} \times 10^{44}\end{array}$ & None & $1.5_{-0.3}^{+0.2} \times 10^{10}$ & $2.0_{-0.4}^{+0.3} \times 10^{28}$ & $\mathrm{~A} 1.6_{-0.5}^{+0.6}$ & A1.6 \\
\hline may 1606 & $\begin{array}{c}3.7_{-0.2}^{+0.3} \\
1.5_{-0.05}^{+0.7} \times 10^{47}\end{array}$ & $\begin{array}{c}8.0_{-0.5}^{+0.1} \\
6.1_{-0.9}^{+2.6} \times 10^{44}\end{array}$ & None & $1.1_{-0.1}^{+0.3} \times 10^{10}$ & $2.1_{-0.3}^{+0.8} \times 10^{28}$ & $\mathrm{~A} 1.7_{-0.4}^{+1.6}$ & A2.3 \\
\hline $\operatorname{may} 1917^{*}$ & $\begin{array}{c}3.2_{-0.03}^{+0.04} \\
3.0_{-0.4}^{+0.4} \times 10^{47}\end{array}$ & $\begin{array}{c}6.4_{-0.1}^{+0.3} \\
6.1_{-2.5}^{+2.4} \times 10^{44}\end{array}$ & None & & & $\mathrm{A} 1.8_{-0.2}^{+0.3}$ & $<\mathrm{A} 1$ \\
\hline
\end{tabular}

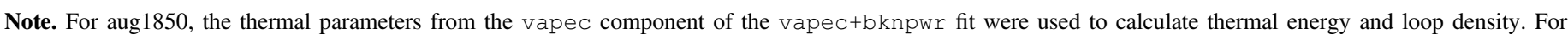
may1917, no obvious thermal volume was seen in a flare time differenced AIA Fe XVIII image, so density and energy estimates have been omitted.

energies of both component thermal models). Thermal energies of each component were calculated assuming an isothermal plasma volume with energy given by

$$
U_{T}=3 k_{B} T \sqrt{\mathrm{EM} f V} \quad[\mathrm{erg}],
$$

where $T$ is the temperature of the plasma, EM is the emission measure, $V$ is the volume, and $f$ is a filling factor (assumed here to be unity). Differenced AIA Fe XVIII images (see Section 4) were used to estimate a volume for each event by considering the geometries of the Fe XVIII loops deemed most likely to be associated with the NuSTAR emission and converting from the resulting area, $A$, to a volume (by taking $A^{3 / 2}$ ). These volumes were additionally used to calculate the density of the thermal plasma in each microflare. Densities are also reported in Table 3, along with the estimated background-subtracted observed GOES class and the expected GOES class calculated from the NuSTAR $T$ and EM using the goes_flux 49.pro IDL routine. The goes_flux 49.pro routine calls CHIANTI version 7.1 and was set to assume coronal abundances (Dere et al. 1997; Landi et al. 2013).

For two more microflares (aug1900 and aug1918), the vapec+vapec models were still unable to fully account for some of the highest-energy emission $(>8 \mathrm{keV}$; see Figure 8 for spectra from aug1900). For both of these events, the vapec +bknpwr models did slightly better at higher energies but found the break energy of the broken power-law distribution to occur between 6 and $7 \mathrm{keV}$, near the strong Fe complex centered around $6.7 \mathrm{keV}$. This weakened the case for the vapec+bknpwr model because at least some of its success could be attributed to the benefit of a break in the spectrum placed near where the thermal continuum is broken by the presence of that Fe complex.

For these two events, it cannot be definitively shown that a nonthermal component is present. Perhaps the high-energy excess above the vapec+vapec fit is the result of a multithermal plasma more complex than that which can be well represented by only two isothermal models. To characterize the flare plasma suggested by the thermal interpretation, the vapec+vapec parameters and thermal energies for these events are reported in Table 3.

For the last event (aug1850), the vapec+vapec fit failed to arrive at a result involving physically realistic plasma temperatures (the higher temperature found was $\sim 4 \times 10^{9} \mathrm{~K}$ ). In this case, the observed NuSTAR spectrum was clearly best fit by the vapec+bknpwr model, as was also found in Glesener et al. (2020). The energy content in nonthermal electrons was calculated by determining the nonthermal power from the bknpwr model parameters (assuming a thick target 

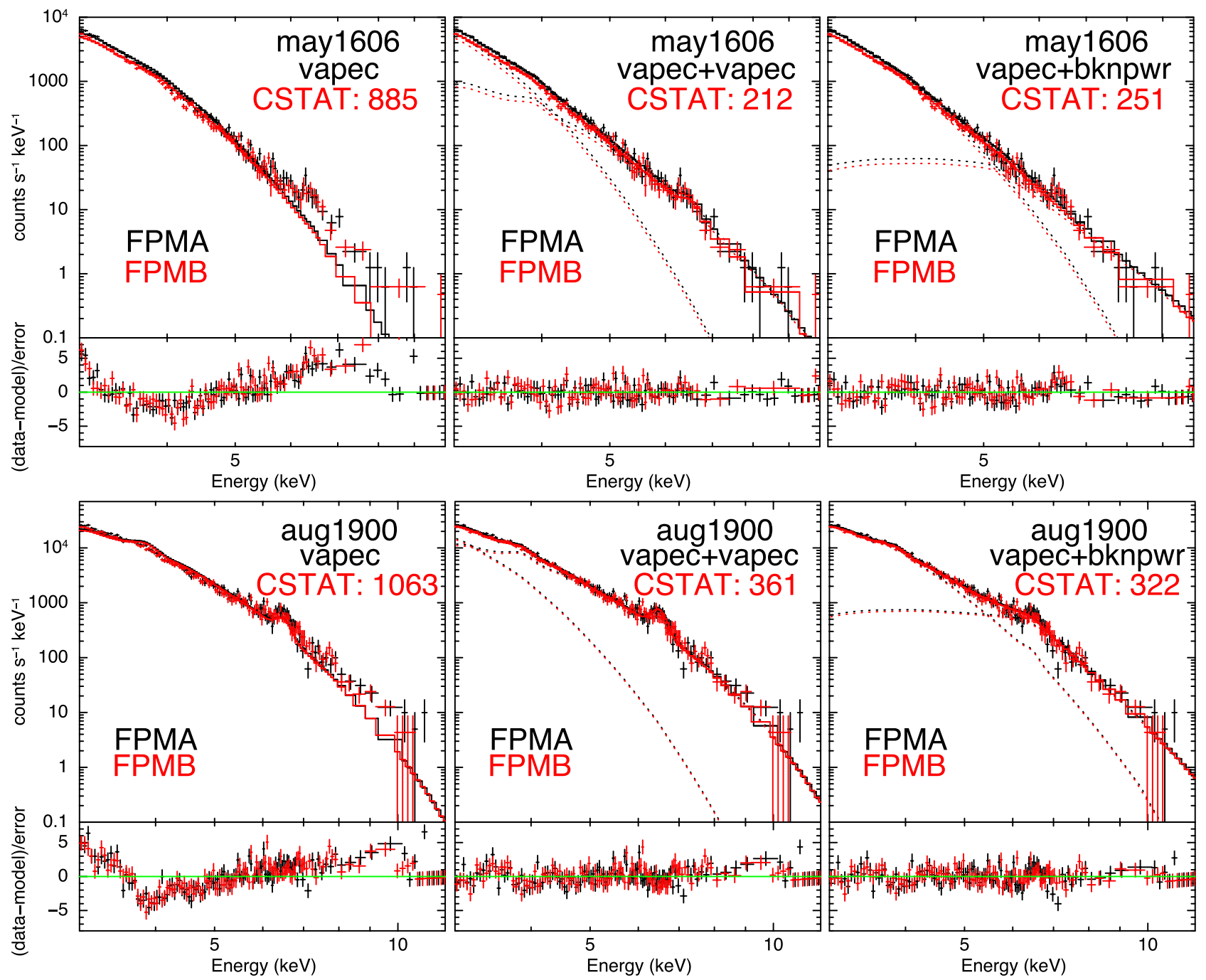

Figure 8. Spectra of may1606 (16:06-16:16 UTC; top row) and aug1900 (19:01-19:08 UTC; bottom row) using three different models: vapec (left), vapec +vapec (middle), and vapec+bknpwr (right). For each example, livetime-corrected FPMA (black) and FPMB (red) count spectra are shown in each top panel, while the bottom panels show the error-normalized residuals. For may1606 (one of the faintest microflares), spectral fits were performed between 3 and 10 keV. The vapec+vapec model provides the strongest fit to the data. The significantly brighter aug1900 was fit over 3-12 keV and required a gain correction (see the Appendix). The slope of the linear gain was freed while performing the vapec+vapec fit, and its resulting value $(0.990)$ was applied as a fixed correction for the vapec and vapec+bknpwr fits. For this event, the vapec+vapec and vapec+bknpwr fits were similar in quality. Thermal fit parameters for both events are reported in Table 3, and vapec+bknpwr parameters are reported for aug1900 in Table 4.

Table 4

Event Parameters (vapec+bknpwr)

\begin{tabular}{|c|c|c|c|c|c|c|c|}
\hline Event & $\begin{array}{c}\text { vapec } \\
T(\mathrm{MK}) \\
\mathrm{EM}\left(\mathrm{cm}^{-3}\right)\end{array}$ & $\begin{array}{l}\text { bknpwr } \\
\text { PhoIndx2 }\end{array}$ & $\begin{array}{c}\text { Break Energy } \\
(\mathrm{keV})\end{array}$ & $\begin{array}{c}\text { Norm. } \\
\left(\frac{\text { photons }}{\mathrm{keV} \mathrm{cm}^{2} \mathrm{~s}} \text { at } 1 \mathrm{keV}\right)\end{array}$ & Gain Shift & $\begin{array}{c}\text { Energy } \\
\text { Nonthermal } \\
(\mathrm{erg}) \\
\end{array}$ & $\begin{array}{c}\text { GOES } \\
\text { Obs. }\end{array}$ \\
\hline aug1918 & $\begin{array}{c}6.4_{-0.04}^{+0.04} \\
4.4_{-0.2}^{+0.2} \times 10^{47}\end{array}$ & $10.0_{-0.5}^{+0.6}$ & $6.4_{-0.1}^{+0.1}$ & $93_{-7}^{+8}$ & $0.984 \pm 0.003$ & $7.7_{-1}^{+1} \times 10^{29}$ & A 4.5 \\
\hline
\end{tabular}

model, as described in Brown 1971) and then integrating over the microflare rise times.

The resulting nonthermal energy is reported in Table 4, along with the vapec+bknpwr model parameters and observed/calculated GOES classes. The vapec component of the vapec+bknpwr model is also shown in Table 3, where it has been used to estimate a thermal energy content and loop density. The resulting thermal and nonthermal parameters, energies, and density were seen to be qualitatively similar to the results of Glesener et al. (2020), though not entirely 
consistent within the uncertainties. The inconsistency is attributed to a slightly different energy range for spectral fitting (3-12 keV here, versus $2.5-12.9 \mathrm{keV}$ ) and a modified gain correction procedure (see the Appendix).

\section{Discussion}

\subsection{Assessment of "Large Flare" Properties in NuSTAR Microflares}

In this section, these NuSTAR microflares are considered with respect to four large flare properties: (1) impulsivity in all HXR energies, (2) earlier peak times in higher-energy emission, (3) greater impulsivity in higher-energy emission, and (4) spatial complexity. The temporal evolution of these microflares shows generally good agreement with the first two of these properties. As shown in Section 3.2, the majority of the events $(8 / 11)$ displayed an impulsive time profile in all NuSTAR energy ranges considered, and all events were either impulsive or at least consistent with an impulsive profile within the uncertainty in the 2-4, 4-6, and 6-8 keV energy ranges. Additionally, 10/11 events (all but may 1917, the faintest microflare) display a trend toward earlier peak times in higherenergy NuSTAR emission. Both of these results provide evidence that the same energy release processes that drive much brighter events may also be at work at the microflare scale.

The third large flare property, greater impulsivity in higherenergy emission, was not observed. However, comparison of impulsivity across energy ranges is complicated by NuSTAR's livetime limitations. Extremely low livetime at microflaring times (combined with proportionally much greater observed flux at the lower end of the NuSTAR energy range) limits the spectral dynamic range (Grefenstette et al. 2016), meaning that higher-energy time profiles have poorer statistics and greater uncertainties in the event asymmetry index (see Table 1). Thus, the failure to confirm this relationship is not seen as proof that it does not exist for these events. Examination of this property in HXR flares at this scale likely requires an HXR instrument with sensitivity similar to NuSTAR that is optimized for the high flux associated with solar observation.

Spatial complexity in HXR emission (the fourth property considered) is a standard feature of larger flares and has also been observed in some RHESSI A- and B-class microflares (e.g., Hannah et al. 2011). Differential centroid locations between differing HXR energy ranges could result from either a thermal plasma with a spatial gradient in temperature or distinct thermal and nonthermal HXR sources. Of these 11 events, there were two microflares for which background subtraction could be performed to isolate flare-specific emission from that of the larger, cooler surrounding active region. These both originated from the same set of loops in AR 12712, and neither displayed differences in the centroids of emission in different energy ranges outside our range of uncertainty. This could imply the observation of emission from cospatial thermal components or, in the nonthermal interpretation, a situation in which loop-top flare-accelerated electrons are thermalized before reaching the loop footpoints (as was concluded in Glesener et al. 2020). A similar lack of spatial complexity was also seen in the NuSTAR microflare examined in Glesener et al. (2017), while, contrastingly, FOXSI HXR microflare emission was shown to be spatially complex in Vievering (2019). Further studies involving a greater number

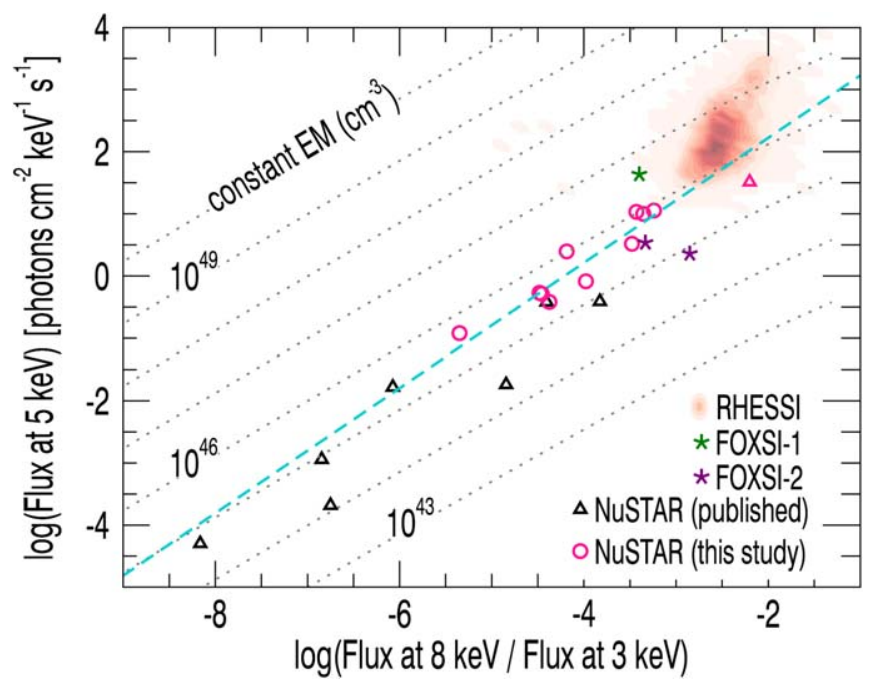

Figure 9. NuSTAR microflares from this study (pink circles) are shown in context with other NuSTAR microflares (black triangles), as well as FOXSI (stars) and RHESSI (red) events (aug1850 is included as a pink triangle to indicate that it is both previously published and a part of this analysis). The vertical axis shows flux at $5 \mathrm{keV}$ (a measure of intensity), while the horizontal axis shows the ratio of flux at 8 and $3 \mathrm{keV}$ (a temperature analog), with reference lines corresponding to constant EM. This allows for a comparison of flaring events that is agnostic to any particular spectral model and shows a strong correlation between these two quantities in the included solar brightenings. A linear fit to the thermal NuSTAR microflares from this paper (excluding aug1850), as well as one additional NuSTAR thermal flare (Glesener et al. 2017), is shown in blue.

of HXR microflares of A class and below are necessary to determine the relative incidence of these two contrasting results and investigate if they are connected to other microflare properties.

\subsection{Thermal versus Nonthermal Interpretation}

As described in Section 5.1, the majority (8/11) of the microflares were found to be best fit by a double thermal model, and the brightest event (aug1850) was found to be best fit by a single thermal model combined with a nonthermal broken power-law distribution. The other two (aug1900 and aug1918) were similarly well fit by both double thermal and thermal + broken power-law models.

To further explore a nonthermal interpretation in those two events, nonthermal energies were calculated from their best-fit broken power-law model components, assuming a thick target model (Brown 1971). These energies are reported in Table 4 along with the thermal and broken power-law model parameters. The nonthermal energies found are around an order of magnitude larger than the derived thermal energies (Table 3). This suggests that, while there is not sufficient spectral evidence to prove the presence of a nonthermal electron distribution in these events, the spectra are consistent with a nonthermal source that could power the observed thermal emission.

Figure 9 shows a brightness-versus-hardness diagram, which includes these 11 microflares in context with previous NuSTAR, FOXSI, and RHESSI events. This representation displays trends in HXR spectral shape across the flares, regardless of their multithermal or nonthermal natures. As discussed in Section 3.3, spectral hardness (defined here as the ratio of fluxes at two HXR energies in the continuum) provides a measure of temperature if the flares are isothermal. This 
Table 5

NuSTAR Microflare Spectral Models and Target IDs

\begin{tabular}{|c|c|c|c|}
\hline Event & Models & & Target ID \\
\hline (Wright et al. 2017) & $\begin{array}{c}\text { VAPEC } \\
T: 4.1_{-0.6}^{+0.2} \mathrm{MK} \\
\text { EM: } 3.1_{-0.8}^{+3.3} \times 10^{46} \mathrm{~cm}^{-3}\end{array}$ & $\begin{array}{c}\text { VAPEC } \\
T: 10.0_{-1.9}^{+0.4} \mathrm{MK} \\
\text { EM: } 1.4_{-0.5}^{+3.7} \times 10^{46} \mathrm{~cm}^{-3}\end{array}$ & SOL2015-04-29T11:29 \\
\hline (Glesener et al. 2017) & $\begin{array}{c}\text { VAPEC }^{*} \\
T: 3.9 \mathrm{MK} \\
\text { EM: } 1.0 \times 10^{46} \mathrm{~cm}^{-3}\end{array}$ & $\begin{array}{c}\text { VAPEC } \\
T: 6.4_{-0.7}^{+0.3} \mathrm{MK} \\
\text { EM: } 2.4_{-0.56}^{+1.49} \times 10^{45} \mathrm{~cm}^{-3}\end{array}$ & SOL2015-09-01T04:00 \\
\hline (Hannah et al. 2019) & $\begin{array}{c}\text { VAPEC }^{*} \\
T: 3.23 \mathrm{MK} \\
\text { EM: } 4.37 \times 10^{46} \mathrm{~cm}^{-3}\end{array}$ & $\begin{array}{c}\text { VAPEC } \\
T: 5.08_{-0.66}^{+0.24} \mathrm{MK} \\
\text { EM: } 6.17_{-1.99}^{+6.80} \times 10^{44} \mathrm{~cm}^{-3}\end{array}$ & SOL2016-07-26T23:35 \\
\hline $\begin{array}{l}\text { (Kuhar et al. 2018) } \\
\text { Flare } 1\end{array}$ & $\begin{array}{c}\text { VAPEC } \\
T: 3.96_{-0.40}^{+0.05} \mathrm{MK} \\
\text { EM: } 8.5_{-0.9}^{+6.3} \times 10^{44} \mathrm{~cm}^{-3}\end{array}$ & & SOL2016-07-26T21:24 \\
\hline Flare 2 & $\begin{array}{c}T: 4.02_{-0.22}^{+0.05} \mathrm{MK} \\
\text { EM: } 1.28_{-0.16}^{+0.44} \times 10^{44} \mathrm{~cm}^{-3}\end{array}$ & & SOL2017-03-21T19:04 \\
\hline Flare 3 & $\begin{array}{c}T: 3.28_{-0.06}^{+0.13} \mathrm{MK} \\
\text { EM: } 5.3_{-1.8}^{+1.8} \times 10^{44} \mathrm{~cm}^{-3}\end{array}$ & & SOL2017-03-21T19:30 \\
\hline $\begin{array}{l}\text { (Glesener et al. 2020) } \\
\text { aug } 1850\end{array}$ & $\begin{array}{l}\text { VAPEC } \\
\text { (Table 4) }\end{array}$ & $\begin{array}{l}\text { BKNPWR } \\
\text { (Table 4) }\end{array}$ & SOL2017-08-21T18:50 \\
\hline $\begin{array}{l}\text { This work } \\
\text { aug } 1900\end{array}$ & $\begin{array}{l}\text { VAPEC } \\
\text { (Table 3) }\end{array}$ & $\begin{array}{l}\text { VAPEC } \\
\text { (Table 3) }\end{array}$ & SOL2017-08-21T19:00 \\
\hline aug1909 & (Table 3) & (Table 3) & SOL2017-08-21T19:09 \\
\hline aug1918 & (Table 3) & (Table 3) & SOL2017-08-21T19:18 \\
\hline may1606 & (Table 3) & (Table 3) & SOL2018-05-29T16:06 \\
\hline may 1618 & (Table 3) & (Table 3) & SOL2018-05-29T16:18 \\
\hline may 1646 & (Table 3) & (Table 3) & SOL2018-05-29T16:46 \\
\hline may 1736 & (Table 3) & (Table 3) & SOL2018-05-29T17:36 \\
\hline may 1747 & (Table 3) & (Table 3) & SOL2018-05-29T17:47 \\
\hline may 1917 & (Table 3) & (Table 3) & SOL2018-05-29T19:17 \\
\hline may 1940 & (Table 3) & (Table 3) & SOL2018-05-29T19:40 \\
\hline (Cooper et al. 2020) & $\begin{array}{c}\text { VAPEC }^{*} \\
T: 3.20 \mathrm{MK} \\
\text { EM: } 1.74 \times 10^{46} \mathrm{~cm}^{-3}\end{array}$ & $\begin{array}{c}\text { VAPEC } \\
T: 6.66_{-0.71}^{+0.69} \mathrm{MK} \\
\text { EM: } 0.80_{-0.32}^{+0.67} \times 10^{44} \mathrm{~cm}^{-3}\end{array}$ & SOL2018-09-09T11:04 \\
\hline
\end{tabular}

Note. Events with target IDs shaded pink were included in the fit line in Figure 9. Thermal models with parameters reported without uncertainty $\left(^{*}\right)$ were fixed thermal background components.

parameterization allows for inclusion of multithermal and nonthermal flares in the visualization. The vertical axis is a measure of intensity at an energy covered by all the instruments in question. For reference, lines of constant EM for an isothermal plasma are overplotted.

Additionally, a blue line shows a linear fit to all of the thermal NuSTAR flares with significant counts at $8 \mathrm{keV}$ (excluding the nonthermal aug1850). These are the brighter events observed; see spectral models and references for all published NuSTAR microflares in Table 5, where events included in this fit are shaded pink. Despite not being included in the fit calculation, aug 1850 lies close to this line, confirming that its spectral shape is not at all unusual when compared to the other flares. This hints that the smaller flares may also have nonthermal aspects that are more challenging to disentangle. In particular, aug1900 is noted as a particularly compelling suspect for a hidden nonthermal component, as it not only showed ambiguity between nonthermal and thermal spectral models but also occurred immediately after aug1850 and from the same set of flare loops within the larger active region (see Figure 7; see also Figure 2 in Glesener et al. 2020).

\section{Conclusions}

In this paper, we have considered 11 NuSTAR microflares, 10 of which are new to the literature, in order to broaden the understanding of the properties of HXR flares at this scale. Consideration of these events together with previous studies 
(Glesener et al. 2017; Wright et al. 2017; Hannah et al. 2019) begins to establish a picture of a "standard" low-A-class HXR microflare. These events commonly display impulsive time profiles, with higher HXR energies peaking before lowerenergy HXRs (and before peaks in lower-energy instruments). Their spectra are dominantly thermal, with flare plasma distributions well approximated by a combination of a brighter, cooler plasma volume $(T=3-5 \mathrm{MK})$ with a fainter, hotter one ( $T=5-10 \mathrm{MK})$.

While the presence of nonthermal emission cannot be definitively established in the majority of cases (the nonthermal behavior of the brightest event, aug1850, remains a singular occurrence among microflares observed so far), the spectra of some of the larger events (aug1900, aug1918) are consistent with a picture involving a nonthermal energy source. Therefore, it seems that the range of magnitudes in peak HXR flux spanned by the microflares observed by NuSTAR so far includes the transition between a regime where nonthermal emission is dominant and one where it is largely indistinguishable from thermal emission. Further exploration of nonthermal properties in HXR events of similar brightness is needed to characterize this transition, which is noted as an especially crucial regime for developing an understanding of particle acceleration at the smallest scales. Such exploration will begin with future NuSTAR microflare observations but will require a solar-dedicated focusing HXR instrument to be approached in a statistical manner.

This work was supported under an NSF Faculty Development Grant (AGS-1429512) to the University of Minnesota, the 2019 NASA Fellowship Program (80NSSC19k1687), an NSF CAREER award (NSF-AGS-1752268), the SolFER DRIVE center (80NSSC20K0627), and the NASA NuSTAR Guest Observer program (80NSSC18K1744). I.G.H is supported by a Royal Society University Fellowship. The authors also wish to thank Pascal Saint-Hilaire for help with eclipse predictions in planning the 2017 August 21 NuSTAR observation.

\section{Appendix Gain Corrections}

For several of the brighter microflares (may1618, may1736, may1747, aug1900, and aug1918), initial spectroscopy using vapec models resulted in features in fit residuals that indicated a systematic failure of these models to accurately fit the NuSTAR spectrum. Specifically, double thermal vapec +vapec models struggled to accurately locate two emission line features present above the thermal continuum (see Figure 10, left).

From the CHIANTI atomic database, we expect the lines in this energy range observed from hot flaring plasma to be a $\mathrm{Ca}$ line at $\sim 3.9 \mathrm{keV}$ ( $\mathrm{Ca}$ XIX) and a complex of lines from transitions in highly ionized Fe centered around $6.7 \mathrm{keV}$ (the Fe XXV resonance line, along with a collection of Fe XXIV satellites; Dere et al. 1997; Del Zanna et al. 2015). When supplied with coronal abundances, the vapec models should be able to accurately represent these features, which are wellunderstood components of emission from solar plasma at coronal temperatures.

The entire catalog of observed NuSTAR microflares was reexamined in XSPEC using a constructed fit designed to examine the handling of these emission lines: a sum of two continuum-only thermal models (nlapec) with two fixed-width Gaussians to simulate line features. The expected 6.7 keV Fe complex was not found in any NuSTAR microflare with sufficient higher-energy statistics to well locate a line in that energy range; in every case, the line feature observed above $6 \mathrm{keV}$ was found to be shifted lower in energy (often to around $6.4 \mathrm{keV}$, a difference far greater than the stated $\sim 40 \mathrm{eV}$ systematic uncertainty in the NuSTAR gain; Madsen et al. 2015).

This includes several events for which simultaneous cospatial emission in the AIA $131 \AA$ channel indicates the presence of plasma at temperatures expected to produce the $\mathrm{Fe}$ complex. Even in an accidentally observed decaying X-class flare (observed at a GOES C-class level by NuSTAR (Grefenstette et al. 2016) and well observed by RHESSI), the higher-energy NuSTAR emission is found to be inconsistent with a $6.7 \mathrm{keV}$ line.

In order to explain these changes in line energy as the result of Doppler shifts, the emitting thermal plasma would need to be traveling toward the Sun at a speed $\left(\sim 10,000 \mathrm{~km} \mathrm{~s}^{-1}\right)$ that is over an order of magnitude larger (and in the wrong direction) than plasma velocities observed in either coronal mass ejections or upward-moving plasma volumes associated with chromospheric evaporation (Gosling et al. 1976; Antonucci et al. 1984).

The possibility of the actual observation of an emission line around $6.4 \mathrm{keV}$ was explored, as $6.39-6.4 \mathrm{keV} \mathrm{Fe} \mathrm{K} \alpha$ emission has been historically observed in $\mathrm{M}$ - and X-class flares by high-resolution spectrometers (interpreted as flaredriven collisional- or photoionization of neutral $\mathrm{Fe}$ in the photosphere; Emslie et al. 1986). This feature has never been identified in an event anywhere close to as faint as these A-class microflares, but the limited spectral resolution ( $\sim 1 \mathrm{keV}$; Lin et al. 2002) of RHESSI and limited sensitivity of other instruments means that such an observation has likely never previously been possible. However, the $\mathrm{Fe} \mathrm{K} \alpha$ explanation for the unexpected incidence of a line at $6.4 \mathrm{keV}$ does not resolve the issue of the failure to observe the $6.7 \mathrm{keV}$ complex even in flares where AIA and RHESSI context imply that it should be observed. Additionally, microflare-driven photospheric $\mathrm{Fe}$ ionization would require a photon or nonthermal electron flux that we do not observe.

An investigation was conducted to determine if this discrepancy could be resolved by adding a correction to the NuSTAR gain. Fitting of response parameters is allowed by XSPEC, specifically the fitting of slope and intercept (offset) parameters describing a linear representation of the gain. Response fitting was performed using standard vapec +vapec models, where both response and model parameters were allowed to vary simultaneously.

With a variation of under $5 \%$ in the value of the gain slope only (with no change to the offset), the vapec+vapec model was able to achieve a dramatically better fit to the NuSTAR spectrum and resolve the line location discrepancy in every event where it was seen. An example is shown in Figure 10, where the addition of only one more parameter (freed gain slope) to the fit allows dramatic improvement in the handling of both observed line features. This event is representative of results in all cases where the line location discrepancy was identified.

The efficacy of this correction across multiple events has led to the conclusion that a small artificial shift in the NuSTAR gain is the most likely explanation for the consistently 

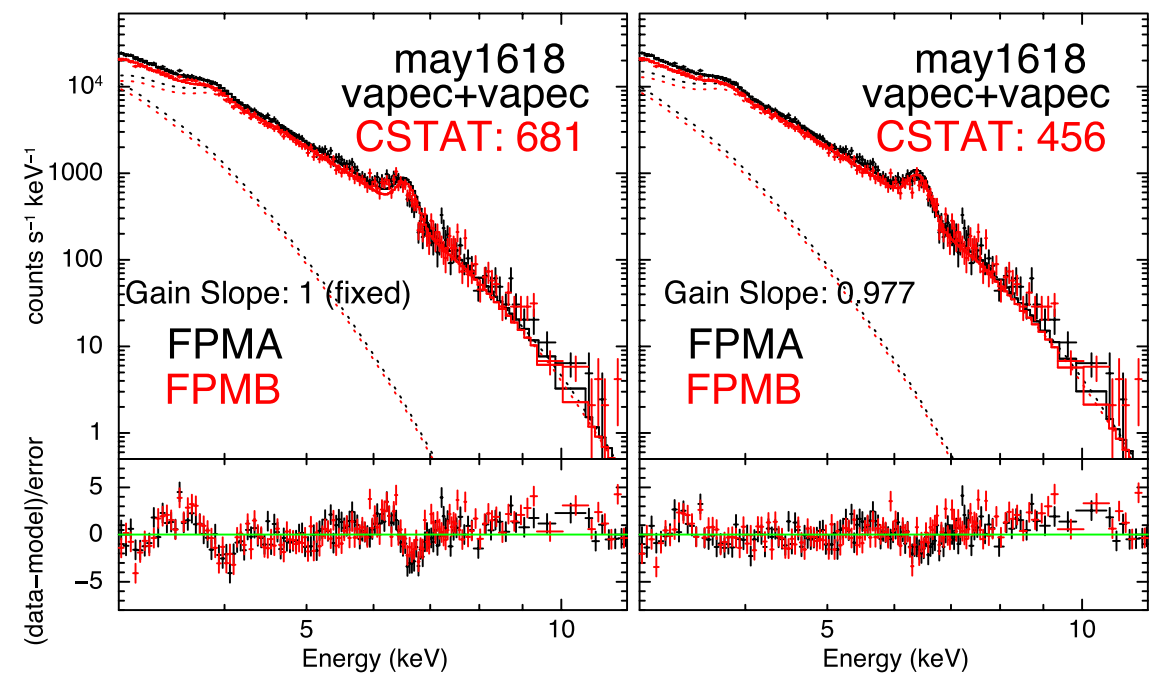

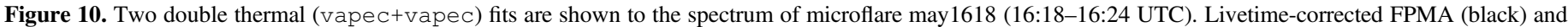

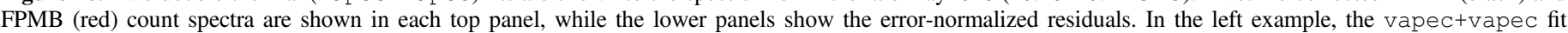

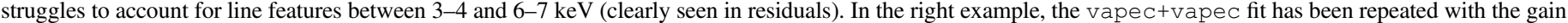
slope parameter freed. Significant improvement is seen in the handling of both emission lines with a small change in gain slope $(<3 \%)$.

identified discrepancies in solar spectral lines. A 5\% gain shift is inconsistent with observations taken in "standard" astrophysical observations, where the sources produce moderate count rates $\left(<1000\right.$ counts $\left.s^{-1}\right)$ and high livetime. See, for example, the joint observation of the neutral Fe $\mathrm{K} \alpha$ complex in Cen A (Fürst et al. 2016). This would also result in an $\sim 4 \mathrm{keV}$ shift of the $86.54 \mathrm{keV}$ 155-Eu calibration line, which is not observed. ${ }^{9}$ We therefore conclude that the extreme count rates $\left(>10^{5}\right.$ counts $\left.\mathrm{s}^{-1}\right)$ and resulting low livetimes $(<1 \%)$ present in many observations of solar active regions and microflares result in a reduced gain of the readout electronics. Such an effect has previously been suggested for the NuSTAR detectors (Bhalerao 2012) but until now has not been observed in any astrophysical sources.

The nature of this problem limits the circumstances in which it can be definitively identified; without any significant line features, the continuum thermal spectrum of a plasma volume with a small gain shift added is indistinguishable from the spectrum of a plasma volume possessing slightly different temperature and EM. It is only when a line is noticeable above the continuum that a gain discrepancy can be readily observed and quantified by its displacement. For NuSTAR solar observations so far, this has occurred only in a livetime regime of $\sim 1 \%$ or below.

The following procedure is prescribed for investigating possible gain shifts when considering low-livetime NuSTAR solar spectra and correcting for them if they are identified. It will be considered a standard aspect of NuSTAR solar spectroscopy moving forward.

1. For events with a line between 6 and $7 \mathrm{keV}$ that can be located at an energy lower than the expected $6.7 \mathrm{keV}$, a gain correction should be found by performing a standard vapec+vapec fit with the gain slope parameter freed.

2. The resulting correction to the gain slope should be applied as a fixed correction for spectral fitting with other model combinations. This is recommended even when vapec+vapec does not give the best fit, as a line

\footnotetext{
9 CAL_NUSTAR_20150316 available at https://heasarc.gsfc.nasa.gov/ docs/heasarc/caldb/nustar/docs/fpm/index.html.
}

location discrepancy can be most accurately identified and corrected for when assuming thermal models only.

3. For events with no noticeable line features (or in the event that a visible line is present with no discrepancy between its expected and observed location), the application of a gain correction is not recommended. If a gain correction is applied, it should be understood that improvement in fit does not, on its own, imply the necessity of a correction. Uncertainty ranges for fit parameters should therefore be extended to include their values when no correction is applied.

In events where a correction is deemed necessary (six of the microflares presented here, as well as other yet-unpublished events), the application of gain corrections according to this method has not been seen to have a dramatic effect on fit parameters. For the six events in this paper, the largest changes in the best-fit parameters were $10 \%$ in temperature and $40 \%$ in EM. ${ }^{10}$ All NuSTAR microflare studies published prior to the identification of this issue have been examined to see if gain corrections should be applied retroactively, and none were found to fit the criteria established here. Therefore, it is not expected that any possible gain discrepancy would have affected those scientific results.

It is noted that the previous paper considering microflare aug1850 (Glesener et al. 2020) was completed at a time when the gain discrepancy had been identified but before this standardized procedure had been established. As such, the gain correction applied in that work was performed by freeing the gain slope during a vapec+bknpwr fit, rather than by the method described here. The qualitative agreement in spectral results for aug 1850 between that paper and this one show that this difference does not affect the earlier conclusions regarding that microflare.

\section{ORCID iDs}

Jessie Duncan (iD https://orcid.org/0000-0002-6872-4406 Lindsay Glesener (iD https://orcid.org/0000-0001-7092-2703

\footnotetext{
${ }^{10}$ Changes in aug1850 bknpwr parameters: break energy, 3\%; photon index 2 , $10 \%$; norm.: $2 \%$.
} 
Brian W. Grefenstette (10 https://orcid.org/0000-0002-1984-2932

Juliana Vievering (1) https://orcid.org/0000-0002-7407-6740

Iain G. Hannah (1) https://orcid.org/0000-0003-1193-8603

David M. Smith (1) https://orcid.org/0000-0002-0542-5759

Säm Krucker (1) https://orcid.org/0000-0002-2002-9180

Stephen M. White (1) https://orcid.org/0000-0002-8574-8629

Hugh Hudson iㅣ https://orcid.org/0000-0001-5685-1283

\section{References}

Antonucci, E., Gabriel, A. H., \& Dennis, B. R. 1984, ApJ, 287, 917

Arnaud, K. A. 1996, in ASP Conf. Ser. 101, XSPEC: The First Ten Years, ed. G. H. Jacoby \& J. Barnes (San Francisco, CA: ASP), 17

Aschwanden, M. J. 2005, Physics of the Solar Corona. An Introduction with Problems and Solutions (2nd ed.; Chichester: Praxis Publishing)

Benz, A. O. 2016, LRSP, 14, 2

Bhalerao, V. 2012, PhD thesis, Caltech, doi:10.7907/CVT1-VR08

Brown, J. C. 1971, SoPh, 18, 489

Cash, W. 1979, ApJ, 228, 939

Christe, S., Hannah, I. G., Krucker, S., McTiernan, J., \& Lin, R. P. 2008, ApJ, 677, 1385

Cooper, K., Hannah, I. G., Grefenstette, B. W., et al. 2020, ApJL, 893, L40

Del Zanna, G. 2013, A\&A, 558, A73

Del Zanna, G., Dere, K. P., Young, P. R., Landi, E., \& Mason, H. E. 2015, A\&A, 582, A56

Dennis, B., Emslie, A., \& Hudson, H. 2011, SSRv, 159, 3

Dennis, B. R., \& Zarro, D. M. 1993, SoPh, 146, 177

Dere, K. P., Landi, E., Mason, H. E., Monsignori Fossi, B. C., \& Young, P. R. 1997, A\&As, 125, 149
Emslie, A. G., Phillips, K. J. H., \& Dennis, B. R. 1986, SoPh, 103, 89 Feldman, U. 1992, PhyS, 46, 202

Fürst, F., Müller, C., Madsen, K. K., et al. 2016, ApJ, 819, 150

Glesener, L., Krucker, S., Christe, S., et al. 2016, Proc. SPIE, 9905, 99050D

Glesener, L., Krucker, S., Duncan, J., et al. 2020, ApJL, 891, L34

Glesener, L., Krucker, S., Hannah, I. G., et al. 2017, ApJ, 845, 122

Gosling, J. T., Hildner, E., MacQueen, R. M., et al. 1976, SoPh, 48, 389

Grefenstette, B. W., Glesener, L., Krucker, S., et al. 2016, ApJ, 826, 20

Hannah, I. G., Christe, S., Krucker, S., et al. 2008, ApJ, 677, 704

Hannah, I. G., Hudson, H. S., Battaglia, M., et al. 2011, SSRv, 159, 263

Hannah, I. G., Kleint, L., Krucker, S., et al. 2019, ApJ, 881, 109

Harrison, F. A., Craig, W. W., Christensen, F. E., et al. 2013, ApJ, 770, 103

Hudson, H. S. 1991, SoPh, 133, 357

Klimchuk, J. A. 2006, SoPh, 234, 41

Kuhar, M., Krucker, S., Glesener, L., et al. 2018, ApJL, 856, L32

Landi, E., Young, P. R., Dere, K. P., Zanna, G. D., \& Mason, H. E. 2013, ApJ, 763,86

Lemen, J. R., Title, A. M., Akin, D. J., et al. 2012, SoPh, 275, 17

Lin, R. P., Dennis, B. R., Hurford, G. J., et al. 2002, SoPh, 210, 3

Madsen, K. K., Harrison, F. A., Markwardt, C. B., et al. 2015, ApJS, 220, 8

Neupert, W. M. 1968, ApJL, 153, L59

Newville, M., Stensitzki, T., Allen, D. B., \& Ingargiola, A. 2014, LMFIT: Non-

Linear Least-Square Minimization and Curve-Fitting for Python, v0.8.0, Zenodo, doi: $10.5281 /$ zenodo. 11813

Parker, E. N. 1988, ApJ, 330, 474

Ryan, D. F., Milligan, R. O., Gallagher, P. T., et al. 2012, ApJS, 202, 11

Temmer, M., Veronig, A., Hanslmeier, A., Otruba, W., \& Messerotti, M. 2002, A\&A, 375, 1049

Vievering, J. T. 2019, PhD thesis, Univ. Minnesota

Warren, H. P., Reep, J. W., Crump, N. A., et al. 2020, ApJ, 896, 51

Wright, P. J., Hannah, I. G., Grefenstette, B. W., et al. 2017, ApJ, 844, 132 